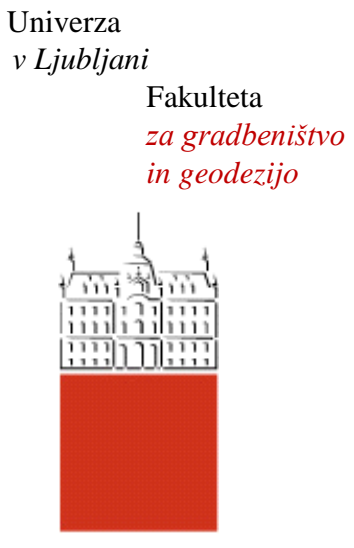

Jamova cesta 2

1000 Ljubljana, Slovenija

http://www3.fgg.uni-lj.si/

\section{DRUGG - Digitalni repozitorij UL FGG http://drugg.fgg.uni-lj.si/}

Ta članek je avtorjeva zadnja recenzirana različica, kot je bila sprejeta po opravljeni recenziji.

Prosimo, da se pri navajanju sklicujte na bibliografske podatke, kot je navedeno:
University
of Ljubljana

Faculty of Civil and Geodetic Engineering

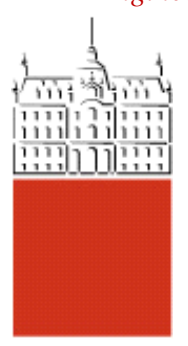

Jamova cesta 2 SI - 1000 Ljubljana, Slovenia http://www3.fgg.uni-lj.si/en/

DRUGG - The Digital Repository http://drugg.fgg.uni-lj.si/

This version of the article is author's manuscript as accepted for publishing after the review process.

When citing, please refer to the publisher's bibliographic information as follows:

Zupan, E., Saje, M., Zupan, D. 2012. Quaternion-based dynamics of geometrically nonlinear spatial beams using the Runge-Kutta method. Finite elements in analysis and design 54: 4860, DOI: $\underline{10.1016 / \text { j.finel.2012.01.007. }}$. 


\title{
Quaternion-based dynamics of geometrically nonlinear spatial beams using the Runge-Kutta method
}

\author{
E. Zupan, M. Saje and D. Zupan* \\ University of Ljubljana, Faculty of Civil and Geodetic Engineering, \\ Jamova 2, SI-1115 Ljubljana, Slovenia
}

\begin{abstract}
The rotational quaternions are the unique four dimensional representation of rotations in three dimensional Euclidean space. In the present paper on the dynamics of non-linear spatial beams, they are used as the basic rotational parameters in formulating the finite-element approach of geometrically exact beam-like structures. The classical concept of parametrizing the rotation matrix by the rotational vector is completely abandoned so that the only rotational parameters are the rotational quaternions representing both rotations and rotational strains in the beam. Because the quaternions are the elements of a four dimensional linear space, their use is an advantage compared to the elements of the special orthogonal group $S O(3)$. This makes possible, e.g. to interpolate the rotational quaternions in a standard additive way and to apply standard Runge-Kutta time integration methods. The present finite-element realization of such a quaternion-based beam theory introduces some further ideas including the use of collocation, the weak form of the consistency equations and the symmetrically integrated boundary conditions which enhances the accuracy or efficiency of the solution and avoids the differential-algebraic type of the discretized governing equations often met in classical formulations. The main advantage of the proposed dynamics formulation is probably its simple implementation into the standard Runge-Kutta procedures. Thus several benefits of the RungeKutta methods such as the absence of the analytical linearization of the discrete governing equations, the local error control and the adaptive time steps are automatically incorporated in the present procedure.
\end{abstract}

Key words: non-linear beam theory, dynamic analysis, finite element method, three-dimensional rotation, quaternion, Runge-Kutta method.

* Corresponding author. E-mail addresss: dejan.zupan@fgg.uni-lj.si 


\section{INTRODUCTION}

In 1986 Simo and Vu-Quoc [29] introduced the first consistent Galerkin-type finite-element formulation of the geometrically exact spatial Reissner beam model. They employed the rotational vector for the parametrization of rotations and used the displacement and rotational vectors as the primary unknowns that needed to be interpolated. Since then a number of innovative formulations have been proposed, which differed in the choice of the parametrization of rotations or in the choice of primary unknowns. For example, Simo et al. [29]-[32], Bottasso and Borri [4], Gosh and Roy [6], Jelenić and Crisfield [17] employed the rotational vector and the displacements as the primary unknowns; Ibrahimbegović [10], [11] introduced a special rotational vector characterized by its additivity; Betsch and Steinmann [3] used the director triad with constraints; Zupan et al. [37] based their implementation on the rotational quaternion; Jelenić and Saje [16] used the rotational vector as the only primary unknown, so that no interpolation of displacements was needed; a formulation where the only primary unknown was the rotational strain measure (the pseudo-curvature) was proposed by Zupan and Saje [34]; their formulation was later on extended to the spatial beam finite element based on the full set of beam strain measures [35].

The fundamental problem of the parametrization of rotations with the rotational vector, particularly in the dynamic analysis, is the singularity of the Rodrigues formula at discrete values, resulting in the singularity of the operator between the rotational vector and the angular velocity. For a long-time dynamic response, one cannot avoid such singularities [36], unless an appropriate time step is used. In contrast, the quaternion parametrization of rotations completely avoids the singularity problem. The use of rotational quaternions in beam and multibody theories has been mostly limited to construct numerically stable and efficient local computational algorithms that avoid singularity problems and accelerate computations (see, e.g. [6], [9], [14], [20], [23], [27], [31]). Zupan and Saje [36] studied the effect of the choice of the parametrization of rotations when integrating rotations from angular velocities using various methods. They have shown that the quaternion parametrization gives more accurate results at the similar computational demands.

The quaternions have only very recently been implemented as the primary unknowns in the static finite-element analysis of the geometrically exact three dimensional beams by Zupan et al. [37] and in the dynamic analysis based on the finite-differences discretization by Lang et al. [22]. Promising results obtained in the above papers motivated us to start formulating a new, quaternion-based finite-element dynamic formulation. As in [22] and [37], we fully abandon the rotation matrix concept and introduce the four-dimensional rotational quaternions as the rotational unknowns of the problem. Thus not only the local com- 
putational algorithms for the rotational vector extraction from the rotation matrix as in, e.g. [30], but also the dynamic equilibrium equations of the beam are written in terms of the quaternions. The strict use of quaternions results in the equations that differ from those found in the classical rotational vectorbased beam formulations. The present space discretization procedure is much alike, but not equal to the one presented in [37], and employs the weak consistency equations at the collocation points as the governing equations of the problem. The formulation employs displacements and rotational quaternions as the interpolated unknowns.

The standard time integrators, including those from the Runge-Kutta families are not appropriate to be used on the special orthogonal group of rotations [32], [36]. In contrast, they are well suited for use, if the rotation is described in the linear four-dimensional quaternion space. In the present paper, the standard Runge-Kutta solver based on the TR-BDF2 method as implemented in Matlab [25] has been used and combined with the present finite-element formulation. Several advantages, such as the local error control and the related adaptive time steps, are standard elements of the solver. The validity of the present formulation is demonstrated by several numerical examples.

\section{GEOMETRY OF THE THREE-DIMENSIONAL BEAM}

The mathematical model of the three dimensional beam consists of the line of centroids, termed the beam axis, and the family of cross-sections, Fig. 1. It is found convenient to introduce two bases: (i) a fixed orthonormal basis $\mathcal{B}_{g}=\left\{\vec{g}_{1}, \vec{g}_{2}, \vec{g}_{3}\right\}$ which, together with a fixed reference point $\mathcal{O}$, defines the global coordinate system $(X, Y, Z)$, and (ii) a local orthonormal basis $\mathcal{B}_{G}=\left\{\vec{G}_{1}, \vec{G}_{2}, \vec{G}_{3}\right\}$. The local basis changes with time and depends on the position of the beam axis in space; together with the reference point on the line of centroids it defines the local coordinate system $(\xi, \eta, \zeta)$. An arbitrary configuration of the three-dimensional beam at time $t$ is uniquely described by the position vector $\vec{r}(x, t), x \in[0, L]$, of the line of centroids and by the family of cross-sections $\mathcal{A}(x, t)$. Here, $x$ is the arc-length parameter of the beam axis in the initial configuration. The family $\mathcal{A}(x, t)$ is related to the reference cross-section $\mathcal{A}_{r}$ placed in plain $Y Z$ with $\mathcal{O}$ being its centroid, and to the family of rotations $\mathcal{R}(x, t)$ that map $\mathcal{A}_{r}$ into $\mathcal{A}(x, t)$ :

$$
\mathcal{R}(x, t): \mathcal{A}_{r} \rightarrow \mathcal{A}(x, t)
$$

The rotational operator $\mathcal{R}(x, t)$ also represents the connection between the global and the local bases 


$$
\begin{aligned}
\mathcal{R}(x, t) & : \mathcal{B}_{g} \rightarrow \mathcal{B}_{G} \\
\vec{G}_{i} & =\mathcal{R} \vec{g}_{i}, \quad i=1,2,3 .
\end{aligned}
$$

We further assume that the cross-sections are rigid, thus conserving their shape during deformation. Therefore the local base vectors $\vec{G}_{2}$ and $\vec{G}_{3}$ are spanning the plane of the cross-section $\mathcal{A}$ and $\vec{G}_{1}$ is its normal vector. Because the family of the local base vectors $\mathcal{B}_{G}$ changes with time, we will also use the term moving basis.

An arbitrary vector, $\vec{a}$, can be expressed with respect to either of the two vector bases

$$
\vec{a}=a_{g 1} \vec{g}_{1}+a_{g 2} \vec{g}_{2}+a_{g 3} \vec{g}_{3}=a_{G 1} \vec{G}_{1}+a_{G 2} \vec{G}_{2}+a_{G 3} \vec{G}_{3}
$$

The components in (1) may also be represented by one-column matrices with three components, $\boldsymbol{a}=\left[\begin{array}{lll}a_{g 1} & a_{g 2} & a_{g 3}\end{array}\right]^{T}$ or $\boldsymbol{a}_{G}=\left[\begin{array}{lll}a_{G 1} & a_{G 2} & a_{G 3}\end{array}\right]^{T}$, where $\boldsymbol{a}$ is the one-column matrix description of a vector $\vec{a}$ with respect to the fixed basis. $\boldsymbol{a}_{G}$ denotes its one-column matrix description with respect to the moving basis. Operator $\mathcal{R}(x, t)$ is a linear operator. Its matrix representation is obtained by expressing local vectors $\vec{G}_{i}$ as the linear combination of the fixed base vectors $\left\{\vec{g}_{1}, \vec{g}_{2}, \vec{g}_{3}\right\}$ :

$$
\begin{aligned}
\vec{G}_{i} & =\mathrm{R}_{1 i} \vec{g}_{1}+\mathrm{R}_{2 i} \vec{g}_{2}+\mathrm{R}_{3 i} \vec{g}_{3}, \quad i=1,2,3 \\
\boldsymbol{R} & =\left[\mathrm{R}_{j i}\right], \quad i, j=1,2,3 .
\end{aligned}
$$

Inserting (2) into (1) gives the matrix relationship between the two one-column representations:

$$
\boldsymbol{a}=\boldsymbol{R} \boldsymbol{a}_{G}
$$

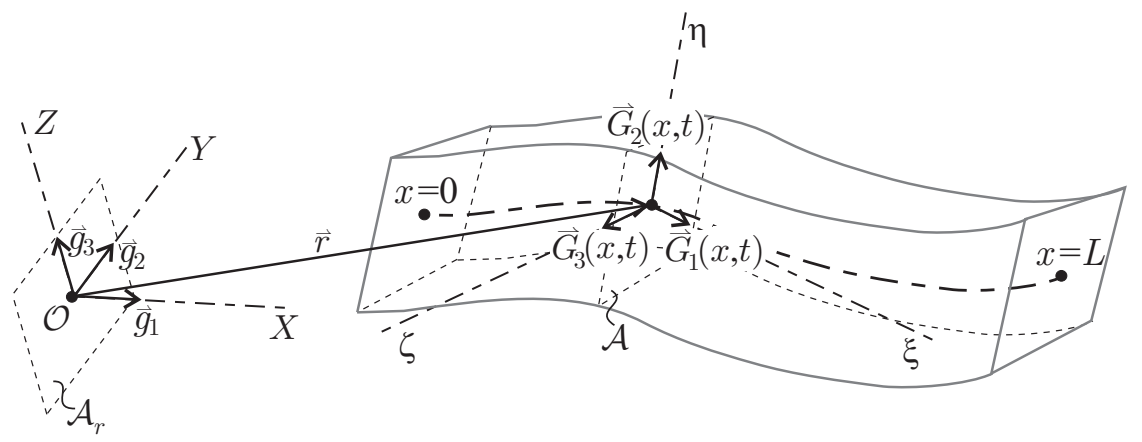

Fig. 1. Model of the three-dimensional beam. 


\section{QUATERNION PARAMETRIZATION OF ROTATIONS}

The rotational operators and rotational quaternions have been described in many publications, see, e.g. [1], [11], [33]. Here only fundamentals of the quaternion algebra will be introduced. For further mathematical details, please consult the monographs by Poreous [26] and Ward [33], and papers by Zupan and Saje [36] and Zupan et al. [37] where the application of the rotational quaternions in the beam theory is given as well.

Quaternions are elements of the four-dimensional Euclidean linear space $\mathbb{R}^{4}$. To distinguish the quaternion (i.e. the four dimensional vector) from the three dimensional vector, we use a hat over the symbol, for example

$$
\begin{aligned}
& \widehat{\boldsymbol{a}}=\left[\begin{array}{llll}
a_{0} & a_{1} & a_{2} & a_{3}
\end{array}\right]^{T} \in \mathbb{R}^{4} \\
& \boldsymbol{a}=\left[\begin{array}{lll}
a_{1} & a_{2} & a_{3}
\end{array}\right]^{T} \in \mathbb{R}^{3} .
\end{aligned}
$$

In quaternions it is a standard practice to separate one dimension from the other three introducing a new notation as

$$
\widehat{\boldsymbol{a}}=a_{0}+\left[\begin{array}{l}
a_{1} \\
a_{2} \\
a_{3}
\end{array}\right]=a_{0}+\boldsymbol{a} .
$$

From (5) it is obvious that $a_{0}$ can be interpreted as the scalar and $\boldsymbol{a}$ as the vector part of the quaternion. The set of quaternions, here marked by $\mathbb{H}$, with elements as defined in Eq. (5), inherits all the operations of $\mathbb{R}^{4}$ (summation $\widehat{\boldsymbol{a}}+\widehat{\boldsymbol{b}}=\left(a_{0}+b_{0}\right)+(\boldsymbol{a}+\boldsymbol{b})$, the scalar multiplication $\lambda\left(a_{0}+\boldsymbol{a}\right)=\lambda a_{0}+\lambda \boldsymbol{a}$ for $\lambda \in \mathbb{R}$, the dot product $\widehat{\boldsymbol{a}} \cdot \widehat{\boldsymbol{b}}=\sum_{i=0}^{3} a_{i} b_{i}$, the Euclidean norm $|\widehat{\boldsymbol{a}}|=\widehat{\boldsymbol{a}} \cdot \widehat{\boldsymbol{a}}$, etc. $)$. We further introduce in $\mathbb{H}$ a special operation called a quaternion multiplication, defined as

$$
\widehat{\boldsymbol{a}} \circ \widehat{\boldsymbol{b}}=\left(a_{0} b_{0}-\boldsymbol{a} \cdot \boldsymbol{b}\right)+\left(b_{0} \boldsymbol{a}+a_{0} \boldsymbol{b}+\boldsymbol{a} \times \boldsymbol{b}\right),
$$

for any $\widehat{\boldsymbol{a}}, \widehat{\boldsymbol{b}} \in \mathbb{H}$. With such a definition of the quaternion product, the space of quaternions $\mathbb{H}$ becomes a non-commutative associative algebra over $\mathbb{R}$. The three-dimensional Euclidean space $\mathbb{R}^{3}$ is a linear subspace in $\mathbb{R}^{4}$ and, thus, a linear subspace in $\mathbb{H}$ :

$$
\boldsymbol{a} \in \mathbb{R}^{3} \Longrightarrow 0+\boldsymbol{a} \in \mathbb{H} .
$$

Any orthonormal basis $\mathcal{B}_{g}=\left\{\vec{g}_{1}, \vec{g}_{2}, \vec{g}_{3}\right\}$ in $\mathbb{R}^{3}$ can be extended to the orthonormal basis $\mathcal{B}_{\widehat{g}}=\left\{\widehat{g}_{0}, \widehat{g}_{1}, \widehat{g}_{2}, \widehat{g}_{3}\right\}$ in $\mathbb{H}$ by the following procedure 


$$
\begin{aligned}
& \widehat{g}_{i}=0+\vec{g}_{i}, \quad i=1,2,3 \\
& \widehat{g}_{0}=1+\overrightarrow{0}=\widehat{1} .
\end{aligned}
$$

The quaternions with a zero scalar part, $0+\boldsymbol{a}$, are called pure quaternions and can be identified with three-dimensional vectors. Finally, we introduce a conjugated quaternion, $\widehat{\boldsymbol{a}}^{*}=a_{0}-\boldsymbol{a}$, where the sign of the vector part is changed.

It is of an utmost importance to understand how the quaternions substitute standard rotation matrices. Let us assume that the rotation is described by the angle of rotation, $\vartheta$, and by the unit vector of the axis of rotation, $\boldsymbol{n}$. Let $\boldsymbol{R}$ denote the corresponding rotation matrix, representing a linear transformation (rotation) in $\mathbb{R}^{3}$ :

$$
\boldsymbol{y}=\boldsymbol{R} \boldsymbol{x} .
$$

Its quaternion representation in $\mathbb{H}$ reads

$$
\widehat{\boldsymbol{y}}=\widehat{\boldsymbol{q}} \circ \widehat{\boldsymbol{x}} \circ \widehat{\boldsymbol{q}}^{*}=\phi_{L}(\widehat{\boldsymbol{q}}) \phi_{R}\left(\widehat{\boldsymbol{q}}^{*}\right) \widehat{\boldsymbol{x}},
$$

where $\widehat{\boldsymbol{x}}$ and $\widehat{\boldsymbol{y}}$ are pure quaternions

$$
\begin{aligned}
& \widehat{\boldsymbol{x}}=0+\boldsymbol{x} \\
& \widehat{\boldsymbol{y}}=0+\boldsymbol{y}
\end{aligned}
$$

matrices $\phi_{L}(\widehat{\boldsymbol{q}})$ and $\phi_{R}\left(\widehat{\boldsymbol{q}}^{*}\right)$ are the component forms of the linear operators of the left and the right quaternion multiplication composed from the components of the quaternion $\widehat{\boldsymbol{q}}$, and

$$
\widehat{\boldsymbol{q}}=\cos \frac{\vartheta}{2}+\boldsymbol{n} \sin \frac{\vartheta}{2}
$$

is a unit quaternion, $|\widehat{\boldsymbol{q}}|=1$, called the rotational quaternion, see [36] or [37] for further details. Note that the rotational quaternion (10) consists of pure geometrical quantities $\vartheta$ and $\boldsymbol{n}$, and thus has a firm geometrical meaning.

When the rotation is position- and/or time-dependent, $\boldsymbol{R}=\boldsymbol{R}(x, t)$, the rotational strain vector, $\vec{\kappa}$, and the angular velocity vector, $\vec{\omega}$, are the measures of the rate of change of rotation with respect to $x$ and $t$, respectively. Both quantities can be expressed with the rotational quaternions via relations

$$
\begin{aligned}
& \widehat{\kappa}=2 \widehat{q}^{\prime} \circ \widehat{q}^{*} \\
& \widehat{\omega}=2 \dot{\widehat{q}} \circ \widehat{q}^{*} .
\end{aligned}
$$

The derivation of Eqs. (11)-(12) is straightforward and can be found in [33] and [37]. Note that both the rotational strain quaternion $\widehat{\kappa}$ and the angular velocity quaternion $\widehat{\omega}$ are pure quaternions with the vector part being identical to the 
standard rotational strain vector and the angular velocity vector, respectively. When expressed with respect to any basis, relations (11)-(12) preserve their simple forms:

$$
\begin{aligned}
\widehat{\boldsymbol{\omega}} & =2 \dot{\hat{\boldsymbol{q}}} \circ \widehat{\boldsymbol{q}}^{*} & \widehat{\boldsymbol{\kappa}} & =2 \widehat{\boldsymbol{q}}^{\prime} \circ \widehat{\boldsymbol{q}}^{*} \\
\widehat{\boldsymbol{\omega}}_{G} & =2 \widehat{\boldsymbol{q}}^{*} \circ \dot{\hat{\boldsymbol{q}}} & \widehat{\boldsymbol{\kappa}}_{G} & =2 \widehat{\boldsymbol{q}}^{*} \circ \widehat{\boldsymbol{q}}^{\prime} .
\end{aligned}
$$

The angular acceleration quaternion is the rate of change of the angular velocity quaternion with respect to time:

$$
\widehat{\alpha}=\dot{\widehat{\omega}}=2 \ddot{\widehat{q}} \circ \widehat{q}^{*}+2 \dot{\widehat{q}} \circ \dot{\widehat{q}}^{*}
$$

The angular acceleration quaternion is also a pure quaternion whose vector part is identical to the standard angular acceleration vector.

\section{RUNGE-KUTTA METHODS}

The Runge-Kutta methods are widely employed in solving systems of ordinary differential equations [5], [7]. They were developed to integrate numerically the initial value problem given by the system of the ordinary first-order differential equations and the initial values

$$
\dot{\boldsymbol{y}}=\boldsymbol{F}(t, \boldsymbol{y}), \quad \boldsymbol{y}\left(t_{0}\right)=\boldsymbol{y}_{0}
$$

The general scheme of the Runge-Kutta methods is given by

$$
\begin{array}{ccc}
\boldsymbol{Y}_{i}\left(t+c_{i} h\right) & =\boldsymbol{y}+\sum_{j=1}^{s} a_{i j} \boldsymbol{F}\left(t+c_{j} h, \boldsymbol{Y}_{j}\left(t+c_{j} h\right)\right) & \sum_{j=1}^{s} a_{i j}=c_{i} \\
\boldsymbol{Y}(t+h)=\boldsymbol{y}+\sum_{j=1}^{s} b_{j} \boldsymbol{F}\left(t+c_{j} h, \boldsymbol{Y}_{j}\left(t+c_{j} h\right)\right) & \sum_{i=1}^{s} b_{i}=1,
\end{array}
$$

where $i=1,2, \ldots, s$, and $s$ is the number of stages, while $a_{i j}, b_{j}$ and $c_{i}$ are the given numerical coefficients which determine the particular member of the Runge-Kutta family. It is well known that most Runge-Kutta methods are not efficient for very stiff problems. Because the equations governing the dynamics of non-linear structures might be stiff even for relatively simple problems, the choice of the particular member of the Runge-Kutta family is crucial.

In the present paper, the second-order Runge-Kutta method ode23tb, as implemented in Matlab [25], has been used. ode23tb is the implementation of the combined trapezoidal rule and the backward differentiation formula, and is known as the TR-BDF2 method [8]. The Butcher array [5] of this method 
reads

$$
\begin{array}{l|llll}
0 & 0 & 0 & 0 & \left(c_{1}=0, a_{11}=a_{12}=a_{13}=0\right) \\
\tau & d & d & 0 & \left(c_{2}=\tau, a_{21}=a_{22}=d, a_{23}=0\right) \\
1 & w & w & d & \left(c_{3}=1, a_{31}=a_{32}=w, a_{33}=d\right) \\
\hline & w & w & d & \left(b_{1}^{2 n d}=b_{2}^{2 n d}=w, b_{3}^{2 n d}=d\right) \\
\hline & \frac{1-w}{3} & \frac{3 w+1}{3} & \frac{d}{3} & \left(b_{1}^{3 r d}=\frac{1-w}{3}, b_{2}^{3 r d}=\frac{3 w+1}{3}, b_{3}^{3 r d}=\frac{d}{3}\right)
\end{array}
$$

where $\tau=2-\sqrt{2}, d=\tau / 2$, and $w=\sqrt{2} / 4$. The fourth line in (17) determines the method of the second order formula, and the fifth one the third order formula. The difference between the solutions obtained by the two formulae enables us to estimate the local error. TR-BDF2 as implemented in Matlab [25] is an attractive implicit one-step method suitable for very stiff problems. Its suitability for very stiff problems derives from an improved local error control procedure which, in an innovative way, considers the stiff components, see [8] for further details. In [8] it is also shown that the method is strongly S-stable and 'optimal in the class of practical one-step methods'. Note that the local error control, despite of its importance, is not a typical characteristic of the methods used in structural dynamics. It is important to stress that the ode23tb needs the Jacobian matrix. To avoid the analytical differentiation, needed in evaluating the Jacobian matrix, its numerical approximation by the finite differences is used as a standard option of the Matlab routines.

\section{EQUATIONS OF THE BEAM}

The complete set of the equations of the three dimensional beam consists of the kinematic equations (20)-(21), the constitutive equations (24)-(25), the equilibrium equations (22)-(23), and the consistency equations (18)-(19):

$$
\begin{aligned}
& \boldsymbol{f}_{1}: \boldsymbol{N}=\boldsymbol{R} \boldsymbol{N}_{G}^{C} \\
& \boldsymbol{f}_{2}: \boldsymbol{M}=\boldsymbol{R} \boldsymbol{M}_{G}^{C} \\
& \boldsymbol{f}_{3}: \boldsymbol{r}^{\prime}=\boldsymbol{R}\left(\gamma_{G}-\boldsymbol{c}_{G}\right) \\
& \boldsymbol{f}_{4}: \boldsymbol{\vartheta}^{\prime}=\boldsymbol{T}^{-1}(\boldsymbol{\vartheta}) \boldsymbol{R}\left(\boldsymbol{\kappa}_{G}-\boldsymbol{d}_{G}\right) \\
& \boldsymbol{f}_{5}: \boldsymbol{n}=-\boldsymbol{N}^{\prime}+\rho A_{r} \ddot{\boldsymbol{r}} \\
& \boldsymbol{f}_{6}: \boldsymbol{m}=-\boldsymbol{M}^{\prime}+\boldsymbol{N} \times \boldsymbol{r}^{\prime}+\boldsymbol{R} \boldsymbol{J}_{\rho} \boldsymbol{R}^{T} \dot{\boldsymbol{\omega}}+\boldsymbol{S}(\boldsymbol{\omega}) \boldsymbol{R} \boldsymbol{J}_{\rho} \boldsymbol{R}^{T} \boldsymbol{\omega} \\
& \boldsymbol{f}_{7}: \boldsymbol{N}_{G}^{C}=\mathcal{C}_{N}\left(\gamma_{G}, \boldsymbol{\kappa}_{G}\right) \\
& \boldsymbol{f}_{8}: \boldsymbol{M}_{G}^{C}=\mathcal{C}_{M}\left(\gamma_{G}, \boldsymbol{\kappa}_{G}\right) .
\end{aligned}
$$

Here, the prime (') denotes the derivative with respect to $x$ and the dot over the symbol $(\cdot)$ denotes the derivative with respect to $t$. The lower index $G$ 
denotes that the vector quantity or the operator is expressed with respect to the local basis. When the fixed basis is used, the index is omitted. The meaning of the notations used in the above equations is described below:

$\boldsymbol{\vartheta}$ standard rotational vector with length $\vartheta$ and direction $\boldsymbol{n}$;

$\boldsymbol{S}(\boldsymbol{a})$ skew-symmetric matrix composed from its axial vector $\boldsymbol{a}, \boldsymbol{S}(\boldsymbol{a}) \boldsymbol{b}=\boldsymbol{a} \times \boldsymbol{b}$;

$\boldsymbol{R}$ rotation matrix, $\boldsymbol{R}=\boldsymbol{I}+\frac{\sin \vartheta}{\vartheta} \boldsymbol{S}(\boldsymbol{\vartheta})+\frac{1-\cos \vartheta}{\vartheta^{2}} \boldsymbol{S}(\boldsymbol{\vartheta}) \boldsymbol{S}(\boldsymbol{\vartheta})$

$\boldsymbol{N}, \boldsymbol{M}$ stress-resultant force and moment vectors at the cross-section;

$\mathcal{C}_{N}, \mathcal{C}_{M}$ operators describing material of the beam;

$\gamma_{G}$ translational strain vector $\left(\gamma_{G 1}\right.$ is the extensional strain, $\gamma_{G 2}, \gamma_{G 3}$ are shear strains);

$\boldsymbol{\kappa}_{G}$ rotational strain vector $\left(\kappa_{G 1}\right.$ is the torsional strain, $\kappa_{G 2}, \kappa_{G 3}$ are the bending strains);

$\boldsymbol{c}_{G}, \boldsymbol{d}_{G}$ variational constants determined from the known strains, position vectors and rotations at the initial configuration;

$\boldsymbol{n}, \boldsymbol{m}$ external distributed force and moment vectors per unit of the undeformed length;

$\boldsymbol{\omega}$ angular velocity;

$\rho$ density (mass per unit initial volume);

$\boldsymbol{J}_{\rho}$ centroidal (mass) inertia matrix of the cross-section;

$\boldsymbol{T}^{-1}$ inverse of the transformation matrix $\boldsymbol{T}$ with respect to the fixed basis, where $\boldsymbol{T}=\boldsymbol{I}+\frac{1-\cos \vartheta}{\vartheta^{2}} \boldsymbol{S}(\boldsymbol{\vartheta})+\frac{\vartheta-\sin \vartheta}{\vartheta^{3}} \boldsymbol{S}(\boldsymbol{\vartheta}) \boldsymbol{S}(\boldsymbol{\vartheta})$.

The solution must also satisfy the boundary conditions

$$
\begin{aligned}
& \boldsymbol{h}_{1}: \boldsymbol{F}^{0}(t)+\boldsymbol{N}(0, t)=\mathbf{0} \\
& \boldsymbol{h}_{2}: \boldsymbol{P}^{0}(t)+\boldsymbol{M}(0, t)=\mathbf{0} \\
& \boldsymbol{h}_{3}: \boldsymbol{F}^{L}(t)-\boldsymbol{N}(L, t)=\mathbf{0} \\
& \boldsymbol{h}_{4}: \boldsymbol{P}^{L}(t)-\boldsymbol{M}(L, t)=\mathbf{0},
\end{aligned}
$$

where $\boldsymbol{F}^{0}, \boldsymbol{P}^{0}, \boldsymbol{F}^{L}, \boldsymbol{P}^{L}$ are the external point forces and moments at the two boundaries, $x=0$ and $x=L$, with respect to the fixed basis. For the dynamic analysis in the quaternion description, we need to prescribe the initial values of the position vector, the rotational quaternion and their time derivatives at $t=0$. We will use the following notations:

$$
\begin{aligned}
\boldsymbol{r}(x, 0) & =\boldsymbol{r}^{[0]}(x) & & \dot{\boldsymbol{r}}(x, 0)=\dot{\boldsymbol{r}}^{[0]}(x) \\
\widehat{\boldsymbol{q}}(x, 0) & =\widehat{\boldsymbol{q}}^{[0]}(x) & & \dot{\boldsymbol{\boldsymbol { q }}}(x, 0)=\dot{\hat{\boldsymbol{q}}}^{[0]}(x) .
\end{aligned}
$$

The details regarding the transformation of the standard rotational vectorbased equations of the static analysis into the quaternion description are presented in [37]. Here we focus only on the transformation of equilibrium equations $(22)-(23)$, because they differ considerably due to inertial terms. Let us introduce some further notations first! 
- Let $\boldsymbol{a}$ be a vector quantity of the beam, then $\widehat{\boldsymbol{a}}$ will represent its pure quaternion extension, i.e. $\widehat{\boldsymbol{a}}=0+\boldsymbol{a}$;

- similarly, $\widehat{\boldsymbol{A}}$ represents a matrix in $\mathbb{R}^{4 \times 4}$ of the form $\left[\begin{array}{ll}1 & \mathbf{0}_{1 \times 3} \\ \mathbf{0}_{3 \times 1} & \boldsymbol{A}\end{array}\right]$ extended from matrix $\boldsymbol{A}$ in $\mathbb{R}^{3 \times 3}$;

- []$_{\mathbb{R}^{3}}$ is the vector part of a pure quaternion, i.e. for $\widehat{\boldsymbol{a}}=0+\boldsymbol{a},[\widehat{\boldsymbol{a}}]_{\mathbb{R}^{3}}=\boldsymbol{a}$.

Note that a skew-symmetric matrix $\boldsymbol{S}(\boldsymbol{a}) \in \mathbb{R}^{3 \times 3}$ when extended according to the above definition to $\widehat{\boldsymbol{S}}(\boldsymbol{a}) \in \mathbb{R}^{4 \times 4}$, represents the standard cross-vector product in the subspace of pure quaternions: $\widehat{\boldsymbol{S}}(\boldsymbol{a}) \widehat{\boldsymbol{b}}=\widehat{\boldsymbol{a} \times \boldsymbol{b}}$. Note also that $\widehat{\boldsymbol{S}}^{T}(\boldsymbol{a})=\widehat{\boldsymbol{S}}(-\boldsymbol{a}) \neq-\widehat{\boldsymbol{S}}(\boldsymbol{a})$ and $\widehat{\boldsymbol{S}}(\boldsymbol{a}) \widehat{\boldsymbol{b}}=\widehat{\boldsymbol{S}}(-\boldsymbol{b}) \widehat{\boldsymbol{a}}$ for any vectors $\boldsymbol{a}, \boldsymbol{b}$.

The transformation into the quaternion description results in four-component equations. In some cases, the additional component of the expanded form represents a trivially satisfied equation $(0=0)$, which has to be removed to avoid possible singularity problems, see Eqs. (32)-(34). However, this is not the case with Eq. (23). An additional scalar equation in Eq. (23) enables us both to treat four scalar components of the rotational quaternion as independent variables and to replace properly the unit norm constraint of the rotational quaternion. The kinematical equation (21) also has a natural 4-component replacement, see Eq. (14).

The equilibrium equations (22)-(23) are transformed in the following manner. Eq. (22) is left unchanged as rotations do not directly appear. In Eq. (23) $\boldsymbol{m}$ and $\boldsymbol{M}$ are expanded to pure quaternions; $\boldsymbol{N} \times \boldsymbol{r}^{\prime}$ is replaced with $\widehat{\boldsymbol{S}}(\boldsymbol{N}) \widehat{\boldsymbol{r}}^{\prime}$; the term $\boldsymbol{R} \boldsymbol{J}_{\rho} \boldsymbol{R}^{T} \dot{\boldsymbol{\omega}}$ is replaced with an equivalent form $\widehat{\boldsymbol{q}} \circ \widehat{\boldsymbol{J}}_{\rho}\left(\widehat{\boldsymbol{q}}^{*} \circ \dot{\hat{\boldsymbol{\omega}}} \circ \widehat{\boldsymbol{q}}\right) \circ \widehat{\boldsymbol{q}}^{*}$, and, analogously, $\boldsymbol{S}(\boldsymbol{\omega}) \boldsymbol{R} \boldsymbol{J}_{\rho} \boldsymbol{R}^{T} \boldsymbol{\omega}$ is replaced with $\widehat{\boldsymbol{S}}(\boldsymbol{\omega})\left(\widehat{\boldsymbol{q}} \circ\left(\widehat{\boldsymbol{J}}_{\rho}\left(\widehat{\boldsymbol{q}}^{*} \circ \widehat{\boldsymbol{\omega}} \circ \widehat{\boldsymbol{q}}\right)\right) \circ \widehat{\boldsymbol{q}}^{*}\right)$, where it was taken into account that the rotation matrix can be described by the left and the right quaternion multiplication, see (8)-(9).

After the complete set of equations of the three dimensional beam has been put into the quaternion representation, it reads:

$$
\begin{aligned}
& \boldsymbol{f}_{1}:\left[\widehat{\boldsymbol{q}} \circ \widehat{\boldsymbol{N}}_{G}^{C} \circ \widehat{\boldsymbol{q}}^{*}\right]_{R^{3}}-\boldsymbol{N}=\mathbf{0} \\
& \boldsymbol{f}_{2}:\left[\widehat{\boldsymbol{q}} \circ \widehat{\boldsymbol{M}}_{G}^{C} \circ \widehat{\boldsymbol{q}}^{*}\right]_{\mathbb{R}^{3}}-\boldsymbol{M}=\mathbf{0} \\
& \boldsymbol{f}_{3}: \boldsymbol{r}^{\prime}-\left[\widehat{\boldsymbol{q}} \circ\left(\widehat{\gamma}_{G}-\widehat{\boldsymbol{c}}_{G}\right) \circ \widehat{\boldsymbol{q}}^{*}\right]_{R^{3}}=\mathbf{0} \\
& \widehat{\boldsymbol{f}}_{4}: 2 \widehat{\boldsymbol{q}}^{*} \circ \widehat{\boldsymbol{q}}^{\prime}-\left(\widehat{\boldsymbol{\kappa}}_{G}-\widehat{\boldsymbol{d}}_{G}\right)=\widehat{\mathbf{0}} \\
& \boldsymbol{f}_{5}: \boldsymbol{N}^{\prime}+\boldsymbol{n}-\rho A_{r} \ddot{\boldsymbol{r}}=\mathbf{0} \\
& \widehat{\boldsymbol{f}}_{6}: \widehat{\boldsymbol{M}}^{\prime}+\widehat{\boldsymbol{m}}-\widehat{\boldsymbol{S}}(\boldsymbol{N}) \widehat{\boldsymbol{r}}^{\prime}-\widehat{\boldsymbol{q}} \circ \widehat{\boldsymbol{J}}_{\rho}\left(\widehat{\boldsymbol{q}}^{*} \circ \dot{\hat{\boldsymbol{\omega}}} \circ \widehat{\boldsymbol{q}}\right) \circ \widehat{\boldsymbol{q}}^{*}
\end{aligned}
$$




$$
\begin{aligned}
&-\widehat{\boldsymbol{S}}(\boldsymbol{\omega})\left(\widehat{\boldsymbol{q}} \circ\left(\widehat{\boldsymbol{J}}_{\rho}\left(\widehat{\boldsymbol{q}}^{*} \circ \widehat{\boldsymbol{\omega}} \circ \widehat{\boldsymbol{q}}\right)\right) \circ \widehat{\boldsymbol{q}}^{*}\right)=\widehat{\mathbf{0}} \\
& \boldsymbol{f}_{7}: \boldsymbol{N}_{G}^{C}-\mathcal{C}_{N}\left(\gamma_{G}, \boldsymbol{\kappa}_{G}\right)=\mathbf{0} \\
& \boldsymbol{f}_{8}: \boldsymbol{M}_{G}^{C}-\mathcal{C}_{M}\left(\gamma_{G}, \boldsymbol{\kappa}_{G}\right)=\mathbf{0} .
\end{aligned}
$$

Notice again that the equations $\widehat{\boldsymbol{f}}_{4}$ and $\widehat{\boldsymbol{f}}_{6}$ represent four-component equations.

When needed, the initial values of the rotational quaternion and its derivative must be obtained from the known initial values of the rotational vector, $\boldsymbol{\vartheta}^{[0]}(x)=\boldsymbol{\vartheta}(x, 0)$, and the angular velocity $\boldsymbol{\omega}^{[0]}(x)=\boldsymbol{\omega}(x, 0)$, using Eqs. (10) and (13):

$$
\begin{aligned}
& \widehat{\boldsymbol{q}}^{[0]}=\cos \frac{\left|\boldsymbol{\vartheta}^{[0]}\right|}{2}+\frac{\boldsymbol{\vartheta}^{[0]}}{\left|\boldsymbol{\vartheta}^{[0]}\right|} \sin \frac{\left|\boldsymbol{\vartheta}^{[0]}\right|}{2} \\
& \dot{\widehat{\boldsymbol{q}}}^{[0]}=\frac{1}{2} \widehat{\boldsymbol{\omega}}^{[0]} \circ \widehat{\boldsymbol{q}}^{[0]} .
\end{aligned}
$$

Equations (34)-(39) and (26)-(29) represent a system of differential-algebraic equations with respect to two parameters, arc-length $x$ and time $t$. Not only the kinematic quantities but also the strains and the internal forces represent the unknowns of the total set of equations. The whole set of equations could be solved as such, but this approach would not be computationally economic. To enhance the computational efficiency, we reduce the number of unknowns as follows. From Eqs. (34)-(35) $\widehat{\boldsymbol{\gamma}}_{G}$ and $\widehat{\boldsymbol{\kappa}}_{G}$ can be uniquely determined for any known pair $\boldsymbol{r}$ and $\widehat{\boldsymbol{q}}$ :

$$
\begin{aligned}
& \widehat{\boldsymbol{\gamma}}_{G}=\widehat{\boldsymbol{q}}^{*} \circ \widehat{\boldsymbol{r}}^{\prime} \circ \widehat{\boldsymbol{q}}+\widehat{\boldsymbol{c}}_{G} \\
& \widehat{\boldsymbol{\kappa}}_{G}=2 \widehat{\boldsymbol{q}}^{*} \circ \widehat{\boldsymbol{q}}^{\prime}+\widehat{\boldsymbol{d}}_{G} .
\end{aligned}
$$

Considering (42) and (43) we express the internal stress resultants $\boldsymbol{N}_{G}^{C}$ and $\boldsymbol{M}_{G}^{C}$ with $\boldsymbol{r}$ and $\widehat{\boldsymbol{q}}$ as prescribed by the constitutive equations and then insert into (32)-(33). In order to keep the terms with the derivatives with respect to time, $\ddot{\boldsymbol{r}}$ and $\dot{\hat{\boldsymbol{\omega}}}$, as simple as possible, we here choose an alternative approach and employ the weak (differentiated) form of the consistency equations (32)(33), so that $\boldsymbol{N}^{\prime}$ and $\boldsymbol{M}^{\prime}$ can be directly expressed from (36)-(37):

$$
\begin{aligned}
\boldsymbol{f}_{1}^{\prime}= & {\left[\widehat{\boldsymbol{q}} \circ \widehat{\mathcal{C}}_{N}\left(\gamma_{G}, \boldsymbol{\kappa}_{G}\right) \circ \widehat{\boldsymbol{q}}^{*}\right]_{R^{3}}^{\prime}+\boldsymbol{n}-\rho A_{r} \ddot{\boldsymbol{r}}=\mathbf{0} } \\
\widehat{\boldsymbol{f}}_{2}^{\prime}= & \left(\widehat{\boldsymbol{q}} \circ \widehat{\mathcal{C}}_{M}\left(\gamma_{G}, \boldsymbol{\kappa}_{G}\right) \circ \widehat{\boldsymbol{q}}^{*}\right)^{\prime}+\widehat{\boldsymbol{m}}-\widehat{\boldsymbol{S}}(\boldsymbol{N}) \widehat{\boldsymbol{r}}^{\prime} \\
& \quad-\widehat{\boldsymbol{q}} \circ\left(\widehat{\boldsymbol{J}}_{\rho}\left(\widehat{\boldsymbol{q}}^{*} \circ \dot{\hat{\boldsymbol{\omega}}} \circ \widehat{\boldsymbol{q}}\right)\right) \circ \widehat{\boldsymbol{q}}^{*}-\widehat{\boldsymbol{S}}(\widehat{\boldsymbol{\omega}})\left(\widehat{\boldsymbol{q}} \circ\left(\widehat{\boldsymbol{J}}_{\rho}\left(\widehat{\boldsymbol{q}}^{*} \circ \widehat{\boldsymbol{\omega}} \circ \widehat{\boldsymbol{q}}\right)\right) \circ \widehat{\boldsymbol{q}}^{*}\right)=\widehat{\mathbf{0}},
\end{aligned}
$$


where $\boldsymbol{\gamma}_{G}$ and $\boldsymbol{\kappa}_{G}$ are given by (42) and (43), and $\widehat{\boldsymbol{\omega}}$ and $\dot{\hat{\boldsymbol{\omega}}}$ by (12) and (15). The boundary conditions (26)-(29) are solved in the strong form. The boundary stress resultants are obtained by integrating (36)-(37):

$$
\begin{aligned}
\boldsymbol{N}(0, t)= & \boldsymbol{N}\left(\frac{L}{2}, t\right)+\int_{0}^{L / 2} \boldsymbol{n} d x-\int_{0}^{L / 2} \rho A_{r} \ddot{\boldsymbol{r}} d x \\
\widehat{\boldsymbol{M}}(0, t)= & \boldsymbol{M}\left(\frac{L}{2}, t\right)+\int_{0}^{L / 2} \widehat{\boldsymbol{m}} d x-\int_{0}^{L / 2} \widehat{\boldsymbol{S}}(\widehat{\boldsymbol{N}}) \widehat{\boldsymbol{r}}^{\prime} d x \\
& -\int_{0}^{L / 2}\left[\widehat{\boldsymbol{q}} \circ \widehat{\boldsymbol{J}}_{\rho}\left(\widehat{\boldsymbol{q}}^{*} \circ \dot{\hat{\boldsymbol{\omega}}} \circ \widehat{\boldsymbol{q}}\right) \circ \widehat{\boldsymbol{q}}^{*}+\widehat{\boldsymbol{S}}(\widehat{\boldsymbol{\omega}})\left(\widehat{\boldsymbol{q}} \circ \widehat{\boldsymbol{J}}_{\rho}\left(\widehat{\boldsymbol{q}}^{*} \circ \widehat{\boldsymbol{\omega}} \circ \widehat{\boldsymbol{q}}\right) \circ \widehat{\boldsymbol{q}}^{*}\right)\right] d x \\
\boldsymbol{N}(L, t)= & \boldsymbol{N}\left(\frac{L}{2}, t\right)-\int_{L / 2}^{L} \boldsymbol{n} d x+\int_{L / 2}^{L} \rho A_{r} \ddot{\boldsymbol{r}} d x \\
\widehat{\boldsymbol{M}}(0, t)= & \boldsymbol{M}\left(\frac{L}{2}, t\right)+\int_{0}^{L / 2} \widehat{\boldsymbol{m}} d x-\int_{0}^{L / 2} \widehat{\boldsymbol{S}}(\widehat{\boldsymbol{N}}) \widehat{\boldsymbol{r}}^{\prime} d x \\
& -\int_{0}^{L / 2}\left[\widehat{\boldsymbol{q}} \circ \widehat{\boldsymbol{J}}_{\rho}\left(\widehat{\boldsymbol{q}}^{*} \circ \dot{\hat{\boldsymbol{\omega}}} \circ \widehat{\boldsymbol{q}}\right) \circ \widehat{\boldsymbol{q}}^{*}+\widehat{\boldsymbol{S}}(\widehat{\boldsymbol{\omega}})\left(\widehat{\boldsymbol{q}} \circ \widehat{\boldsymbol{J}}_{\rho}\left(\widehat{\boldsymbol{q}}^{*} \circ \widehat{\boldsymbol{\omega}} \circ \widehat{\boldsymbol{q}}\right) \circ \widehat{\boldsymbol{q}}^{*}\right)\right] d x .
\end{aligned}
$$

As observed above, the boundary values are expressed with respect to the midpoint of the beam to conserve the symmetric form of equations. After $\boldsymbol{N}\left(\frac{L}{2}, t\right)=\boldsymbol{N}^{L / 2}$ and $\boldsymbol{M}\left(\frac{L}{2}, t\right)=\boldsymbol{M}^{L / 2}$ are evaluated from the constitutive equations, we finally have

$$
\begin{aligned}
\boldsymbol{h}_{1}: & \widehat{\boldsymbol{S}}^{0}+\left[\widehat{\boldsymbol{q}}^{L / 2} \circ \widehat{\mathcal{C}}_{N}^{L / 2}\left(\boldsymbol{\gamma}_{G}, \boldsymbol{\kappa}_{G}\right) \circ \widehat{\boldsymbol{q}}^{L / 2 *}\right]_{\mathbb{R}^{3}}+\int_{0}^{L / 2} \boldsymbol{n} d x-\int_{0}^{L / 2} \rho A_{r} \ddot{\boldsymbol{r}} d x=\mathbf{0} \\
\widehat{\boldsymbol{h}}_{2}: & \widehat{\boldsymbol{P}}^{0}+\left[\widehat{\boldsymbol{q}}^{L / 2} \circ \widehat{\mathcal{C}}_{M}^{L / 2}\left(\boldsymbol{\gamma}_{G}, \boldsymbol{\kappa}_{G}\right) \circ \widehat{\boldsymbol{q}}^{L / 2 *}\right]_{\mathbb{R}^{3}}+\int_{0}^{L / 2} \widehat{\boldsymbol{m}} d x-\int_{0}^{L / 2} \widehat{\boldsymbol{S}}(\widehat{\boldsymbol{N}}) \widehat{\boldsymbol{r}}^{\prime} d x \\
& -\int_{0}^{L / 2} \widehat{\boldsymbol{q}} \circ \widehat{\boldsymbol{J}}_{\rho}\left(\widehat{\boldsymbol{q}}^{*} \circ \dot{\hat{\boldsymbol{\omega}}} \circ \widehat{\boldsymbol{q}}\right) \circ \widehat{\boldsymbol{q}}^{*} d x-\int_{0}^{L / 2} \widehat{\boldsymbol{S}}(\widehat{\boldsymbol{\omega}})\left(\widehat{\boldsymbol{q}} \circ \widehat{\boldsymbol{J}}_{\rho}\left(\widehat{\boldsymbol{q}}^{*} \circ \widehat{\boldsymbol{\omega}} \circ \widehat{\boldsymbol{q}}\right) \circ \widehat{\boldsymbol{q}}^{*}\right) d x=\widehat{\mathbf{0}}
\end{aligned}
$$

$$
\boldsymbol{h}_{3}: \boldsymbol{S}^{L}-\left[\widehat{\boldsymbol{q}}^{L / 2} \circ \widehat{\mathcal{C}}_{N}^{L / 2}\left(\boldsymbol{\gamma}_{G}, \boldsymbol{\kappa}_{G}\right) \circ \widehat{\boldsymbol{q}}^{L / 2 *}\right]_{\mathbb{R}^{3}}+\int_{L / 2}^{L} \boldsymbol{n} d x-\int_{L / 2}^{L} \rho A_{r} \ddot{\boldsymbol{r}} d x=\mathbf{0}
$$




$$
\begin{aligned}
\widehat{\boldsymbol{h}}_{4} & : \widehat{\boldsymbol{P}}^{L}-\left[\widehat{\boldsymbol{q}}^{L / 2} \circ \widehat{\mathcal{C}}_{M}^{L / 2}\left(\boldsymbol{\gamma}_{G}, \boldsymbol{\kappa}_{G}\right) \circ \widehat{\boldsymbol{q}}^{L / 2 *}\right]_{\mathbb{R}^{3}}+\int_{L / 2}^{L} \widehat{\boldsymbol{m}} d x-\int_{L / 2}^{L} \widehat{\boldsymbol{S}}(\widehat{\boldsymbol{N}}) \widehat{\boldsymbol{r}}^{\prime} d x \\
& -\int_{L / 2}^{L} \widehat{\boldsymbol{q}} \circ\left(\widehat{\boldsymbol{J}}_{\rho}\left(\widehat{\boldsymbol{q}}^{*} \circ \dot{\hat{\boldsymbol{\omega}}} \circ \widehat{\boldsymbol{q}}\right)\right) \circ \widehat{\boldsymbol{q}}^{*} d x-\int_{L / 2}^{L} \widehat{\boldsymbol{S}}(\widehat{\boldsymbol{\omega}})\left(\widehat{\boldsymbol{q}} \circ \widehat{\boldsymbol{J}}_{\rho}\left(\widehat{\boldsymbol{q}}^{*} \circ \widehat{\boldsymbol{\omega}} \circ \widehat{\boldsymbol{q}}\right) \circ \widehat{\boldsymbol{q}}^{*}\right) d x=\widehat{\mathbf{0}} .
\end{aligned}
$$

Eqs. (44)-(49) represent the final form of the governing equations. Note that the present approach results in the boundary equations being the differential rather than the algebraic equations with respect to time. In such a way we have avoided solving the system of differential-algebraic equations.

\section{NUMERICAL SOLUTION}

In formulating the numerical method for the solution, primary unknowns $\boldsymbol{r}(x, t)$ and $\widehat{\boldsymbol{q}}(x, t)$ are replaced by a set of one-parameter functions $\boldsymbol{r}^{p}(t)=$ $\boldsymbol{r}\left(x_{p}, t\right)$ and $\widehat{\boldsymbol{q}}^{p}(t)=\widehat{\boldsymbol{q}}\left(x_{p}, t\right)$ at $N+2$ discretization points $x_{p} ; p=0, \ldots$, $N+1$, chosen from the interval $[0, L]$ so that $x_{0}=0$ and $x_{N+1}=L$. It is suitable to introduce an interpolation of these functions with respect to parameter $x$ as

$$
\boldsymbol{r}(x, t)=\sum_{p=0}^{N+1} L_{p}(x) \boldsymbol{r}^{p}(t) \quad \widehat{\boldsymbol{q}}(x, t)=\sum_{p=0}^{N+1} L_{p}(x) \widehat{\boldsymbol{q}}^{p}(t) .
$$

$L_{p}(x)$ are interpolation functions. In the present paper, the Lagrangian polynomials of degree $N+1$ are applied. The discretization of the governing equations needs to be performed in accord with the discretization of the unknown quantities. Here we choose the collocation method and require that Eqs. (44)(45) are satisfied at $N$ pre-selected collocation points, $\eta_{k} ; k=1, \ldots, N$. For the sake of simplicity and efficiency, they are taken to coincide with the internal discretization points, $x_{k} ; k=1, \ldots, N$. The discretization with respect to $x$ as described above results in a system of $7(N+2)$ second-order scalar differential equations for $7(N+2)$ unknown components of $\boldsymbol{r}^{p}(t)$ and $\widehat{\boldsymbol{q}}^{p}(t)$. As parameter $x$ has now been eliminated by the discretization, the only independent parameter left in the discretized equations is time $t$. Further, we transform the resulting system of the second-order differential equations into the system of the first order. To this end we introduce additional unknowns the first derivatives of the unknown functions at points $x_{p}, p=0,1, \ldots, N+1$ :

$$
\boldsymbol{v}^{p}(t)=\dot{\boldsymbol{r}}^{p}(t) \quad \widehat{\boldsymbol{\lambda}}^{p}(t)=\dot{\widehat{\boldsymbol{q}}}^{p}(t),
$$


representing velocities and quaternion velocities. The interpolation of the additional unknowns with respect to $x$ follows directly from (50):

$$
\boldsymbol{v}(x, t)=\sum_{p=0}^{N+1} L_{p}(x) \boldsymbol{v}^{p}(t) \quad \hat{\boldsymbol{\lambda}}(x, t)=\sum_{p=0}^{N+1} L_{p}(x) \widehat{\boldsymbol{\lambda}}^{p}(t) .
$$

In a sharp contrast to the classical approach, where the angular velocity $\boldsymbol{\omega}$ plays the role of the basic unknown, the first derivative of the rotational quaternion with respect to time here becomes an additional unknown. As $\dot{\widehat{\boldsymbol{q}}}$ is directly related to $\widehat{\boldsymbol{\omega}}$

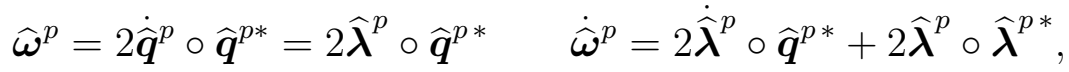

we have no trouble to rewrite the discretized equations in terms of the chosen set of unknown functions. In order to employ the standard Runge-Kutta solvers, we need to rewrite the beam equations (46)-(49) into the following standard form

$$
\boldsymbol{M}(\boldsymbol{y}, t) \dot{\boldsymbol{y}}=\boldsymbol{F}(\boldsymbol{y}, t),
$$

where $\boldsymbol{y}$ is the vector of the unknown variables, $\boldsymbol{F}$ is the right-hand side dependent only on $t$ and $\boldsymbol{y}$, and $\boldsymbol{M}(\boldsymbol{y}, t)$ is the time and state dependent mass matrix. To that end the left-hand side of the beam equations should be expressed as the linear combination of the first derivatives, whose coefficients form the mass matrix. After Eqs. (44)-(49) are discretized and Eq. (51) is used along with relation (53), $\dot{\hat{\boldsymbol{\lambda}}}^{k}$ appears only in the terms $\widehat{\boldsymbol{q}}^{k} \circ \widehat{\boldsymbol{J}}_{\rho}\left(\widehat{\boldsymbol{q}}^{k *} \circ 2 \dot{\hat{\boldsymbol{\lambda}}}^{k}\right) \circ \widehat{\boldsymbol{q}}^{k *}$ containing the quaternion product. To obtain the equivalent expression in terms of the four-dimensional linear algebra, the matrix form of the left and the right quaternion multiplication (see Eq. (9)) must be employed:

$$
\widehat{\boldsymbol{q}}^{k} \circ \widehat{\boldsymbol{J}}_{\rho}\left(\widehat{\boldsymbol{q}}^{k *} \circ 2 \dot{\hat{\boldsymbol{\lambda}}}^{k}\right) \circ \widehat{\boldsymbol{q}}^{k *}=2 \boldsymbol{\phi}_{L}\left(\widehat{\boldsymbol{q}}^{k}\right) \boldsymbol{\phi}_{R}\left(\widehat{\boldsymbol{q}}^{k *}\right) \widehat{\boldsymbol{J}}_{\rho} \boldsymbol{\phi}_{L}\left(\widehat{\boldsymbol{q}}^{k *}\right) \dot{\hat{\boldsymbol{\lambda}}}^{k}
$$

The term $\phi_{L}\left(\widehat{\boldsymbol{q}}^{k}\right) \boldsymbol{\phi}_{R}\left(\widehat{\boldsymbol{q}}^{k *}\right) \widehat{\boldsymbol{J}}_{\rho} \boldsymbol{\phi}_{L}\left(\widehat{\boldsymbol{q}}^{k *}\right)$ represents the matrix product of fourdimensional matrices and is thus the four-dimensional matrix, which is well in accordance with the general form (54). After considering Eqs. (51), (53), (55), and rearranging some terms, the final form of the discrete governing equations of the beam reads

$$
\begin{aligned}
\boldsymbol{f}_{11}^{k \prime} & : \dot{\boldsymbol{r}}^{k}=\boldsymbol{v}^{k} \\
\boldsymbol{f}_{12}^{k \prime} & : \rho A_{r} \dot{\boldsymbol{v}}^{k} \\
& =\left[\widehat{\boldsymbol{q}}^{k \prime} \circ \widehat{\mathcal{C}}_{N}^{k} \circ \widehat{\boldsymbol{q}}^{k *}+\widehat{\boldsymbol{q}}^{k} \circ \widehat{\mathcal{C}}_{N}^{\prime k} \circ \widehat{\boldsymbol{q}}^{k *}+\widehat{\boldsymbol{q}}^{k} \circ \widehat{\mathcal{C}}_{N}^{k} \circ \widehat{\boldsymbol{q}}^{k * \prime}\right]_{\mathbb{R}^{3}}+\boldsymbol{n}^{k} \\
\widehat{\boldsymbol{f}}_{21}^{k \prime} & : \dot{\boldsymbol{\boldsymbol { q }}}^{k}=\widehat{\boldsymbol{\lambda}}^{k} \\
\widehat{\boldsymbol{f}}_{22}^{k \prime} & : 2 \boldsymbol{\phi}_{L}\left(\widehat{\boldsymbol{q}}^{k}\right) \boldsymbol{\phi}_{R}\left(\widehat{\boldsymbol{q}}^{k *}\right) \widehat{\boldsymbol{J}}_{\rho} \boldsymbol{\phi}_{L}\left(\widehat{\boldsymbol{q}}^{k *}\right) \dot{\hat{\boldsymbol{\lambda}}}^{k} \\
& =\widehat{\boldsymbol{q}}^{k \prime} \circ \widehat{\mathcal{C}}_{M}^{k} \circ \widehat{\boldsymbol{q}}^{k *}+\widehat{\boldsymbol{q}}^{k} \circ \widehat{\mathcal{C}}_{M}^{k \prime} \circ \widehat{\boldsymbol{q}}^{k *}+\widehat{\boldsymbol{q}}^{k} \circ \widehat{\mathcal{C}}_{M}^{k} \circ \widehat{\boldsymbol{q}}^{k * \prime}
\end{aligned}
$$




$$
\begin{aligned}
& +\widehat{\boldsymbol{m}}^{k}-\widehat{\boldsymbol{S}}\left(\widehat{\boldsymbol{q}}^{k} \circ \widehat{\mathcal{C}}_{N}^{k} \circ \widehat{\boldsymbol{q}}^{k *}\right) \widehat{\boldsymbol{r}}^{k \prime}-2 \widehat{\boldsymbol{q}}^{k} \circ \widehat{\boldsymbol{J}}_{\rho}\left(2 \widehat{\boldsymbol{q}}^{k *} \circ \widehat{\boldsymbol{\lambda}}^{k} \circ \widehat{\boldsymbol{\lambda}}^{k *} \circ \widehat{\boldsymbol{q}}^{k}\right) \circ \widehat{\boldsymbol{q}}^{k *} \\
& -4 \widehat{\boldsymbol{S}}\left(\widehat{\boldsymbol{\lambda}}^{k} \circ \widehat{\boldsymbol{q}}^{k *}\right)\left(\widehat{\boldsymbol{q}}^{k} \circ \widehat{\boldsymbol{J}}_{\rho}\left(\widehat{\boldsymbol{q}}^{k *} \circ \hat{\boldsymbol{\lambda}}^{k}\right) \circ \widehat{\boldsymbol{q}}^{k *}\right) .
\end{aligned}
$$

The corresponding boundary equations are

$$
\begin{aligned}
& \boldsymbol{h}_{11}: \dot{\boldsymbol{u}}^{0}=\boldsymbol{v}^{0} \\
& \boldsymbol{h}_{12}: \frac{\rho A_{r} L}{2} \dot{\boldsymbol{v}}^{0}=\boldsymbol{F}^{0}+\boldsymbol{N}^{C, L / 2}+\int_{0}^{L / 2} \boldsymbol{n} d x \\
& \widehat{\boldsymbol{h}}_{21}: \dot{\widehat{\boldsymbol{q}}}^{0}(t)=\widehat{\boldsymbol{\lambda}}^{0}(t) \\
& \widehat{\boldsymbol{h}}_{22}: \int_{0}^{L / 2} 2 \phi_{L}(\widehat{\boldsymbol{q}}) \phi_{R}\left(\widehat{\boldsymbol{q}}^{*}\right) \widehat{\boldsymbol{J}}_{\rho} \boldsymbol{\phi}_{L}\left(\widehat{\boldsymbol{q}}^{*}\right) d x \dot{\hat{\boldsymbol{\lambda}}}^{0}(t) \\
& =\widehat{\boldsymbol{P}}^{0}+\widehat{\boldsymbol{q}}^{L / 2} \circ \widehat{\mathcal{C}}_{M}^{L / 2} \circ \widehat{\boldsymbol{q}}^{L / 2 *}+\int_{0}^{L / 2} \widehat{\boldsymbol{m}} d x-\int_{0}^{L / 2} \widehat{\boldsymbol{S}}\left(\widehat{\boldsymbol{q}} \circ \widehat{\mathcal{C}}_{N} \circ \widehat{\boldsymbol{q}}^{*}\right) \widehat{\boldsymbol{r}}^{\prime} d x \\
& -2 \int_{0}^{L / 2} \widehat{\boldsymbol{q}} \circ\left(\widehat{\boldsymbol{J}}_{\rho}\left(2 \widehat{\boldsymbol{q}}^{*} \circ \widehat{\boldsymbol{\lambda}} \circ \widehat{\boldsymbol{\lambda}}^{*} \circ \widehat{\boldsymbol{q}}\right)\right) \circ \widehat{\boldsymbol{q}}^{*} d x \\
& -4 \int_{0}^{L / 2} \widehat{\boldsymbol{S}}\left(\widehat{\boldsymbol{\lambda}} \circ \widehat{\boldsymbol{q}}^{*}\right)\left(\widehat{\boldsymbol{q}}^{k} \circ\left(\widehat{\boldsymbol{J}}_{\rho}\left(\widehat{\boldsymbol{q}}^{*} \circ \widehat{\boldsymbol{\lambda}}\right)\right) \circ \widehat{\boldsymbol{q}}^{*}\right) d x \\
& \boldsymbol{h}_{31}: \dot{\boldsymbol{u}}^{L}=\boldsymbol{v}^{L} \\
& \boldsymbol{h}_{32}: \frac{\rho A_{r} L}{2} \dot{\boldsymbol{v}}^{L}=\boldsymbol{F}^{L}-\boldsymbol{N}^{C, L / 2}+\int_{L / 2}^{L} \boldsymbol{n} d x \\
& \widehat{\boldsymbol{h}}_{41}: \dot{\widehat{\boldsymbol{q}}}^{L}=\widehat{\boldsymbol{\lambda}}^{L} \\
& \widehat{\boldsymbol{h}}_{42}: \int_{L / 2}^{L} 2 \boldsymbol{\phi}_{L}(\widehat{\boldsymbol{q}}) \boldsymbol{\phi}_{R}\left(\widehat{\boldsymbol{q}}^{*}\right) \widehat{\boldsymbol{J}}_{\rho} \boldsymbol{\phi}_{L}\left(\widehat{\boldsymbol{q}}^{*}\right) d x \dot{\hat{\boldsymbol{\lambda}}}^{L} \\
& =\widehat{\boldsymbol{P}}^{L}-\widehat{\boldsymbol{q}}^{L / 2} \circ \widehat{\mathcal{C}}_{M}^{L / 2} \circ \widehat{\boldsymbol{q}}^{L / 2 *}+\int_{L / 2}^{L} \widehat{\boldsymbol{m}} d x-\int_{L / 2}^{L} \widehat{\boldsymbol{S}}\left(\widehat{\boldsymbol{q}} \circ \widehat{\mathcal{C}}_{N} \circ \widehat{\boldsymbol{q}}^{*}\right) \widehat{\boldsymbol{r}}^{\prime} d x \\
& -2 \int_{L / 2}^{L} \widehat{\boldsymbol{q}} \circ\left(\widehat{\boldsymbol{J}}_{\rho}\left(2 \widehat{\boldsymbol{q}}^{*} \circ \hat{\boldsymbol{\lambda}} \circ \hat{\boldsymbol{\lambda}}^{*} \circ \widehat{\boldsymbol{q}}\right)\right) \circ \widehat{\boldsymbol{q}}^{*} d x \\
& -4 \int_{L / 2}^{L} \widehat{\boldsymbol{S}}\left(\widehat{\boldsymbol{\lambda}} \circ \widehat{\boldsymbol{q}}^{*}\right)\left(\widehat{\boldsymbol{q}} \circ\left(\widehat{\boldsymbol{J}}_{\rho}\left(\widehat{\boldsymbol{q}}^{*} \circ \hat{\boldsymbol{\lambda}}\right)\right) \circ \widehat{\boldsymbol{q}}^{*}\right) d x .
\end{aligned}
$$

The integrals appearing in the above equations are evaluated numerically using the Gaussian integration method. 
Note that the method ode23tb as implemented in Matlab does not automatically conserve the unit norm of the rotational quaternion. In order to obtain the kinematically admissible results, the rotational quaternions are normalized after each time step has been completed. An alternative Runge-Kutta method that inherently conserves the unity of the rotational quaternion is not the subject of the present paper.

\section{NUMERICAL EXAMPLES}

The numerical examples will demonstrate the validity, performance and accuracy of the proposed finite displacement and rotation formulation. Numerical solutions were obtained in the computing environment Matlab [25]. Here we limit our studies to linear elastic materials with the diagonal form of operators $\mathcal{C}_{N}$ and $\mathcal{C}_{M}$ :

$$
\begin{aligned}
\mathcal{C}_{N}\left(\boldsymbol{\gamma}_{G}, \boldsymbol{\kappa}_{G}\right) & =\left[\begin{array}{ccc}
E A_{1} & 0 & 0 \\
0 & G A_{2} & 0 \\
0 & 0 & G A_{3}
\end{array}\right] \boldsymbol{\gamma}_{G} \\
\mathcal{C}_{M}\left(\boldsymbol{\gamma}_{G}, \boldsymbol{\kappa}_{G}\right) & =\left[\begin{array}{ccc}
G J_{1} & 0 & 0 \\
0 & E J_{2} & 0 \\
0 & 0 & E J_{3}
\end{array}\right] \boldsymbol{\kappa}_{G} .
\end{aligned}
$$

$E$ and $G$ denote elastic and shear moduli of material; $A_{1}$ is the cross-sectional area; $J_{1}$ is the torsional inertial moment of the cross-section; $A_{2}$ and $A_{3}$ are the effective shear areas in the principal inertial directions $\vec{G}_{2}$ and $\vec{G}_{3}$ of the cross-section; $J_{2}$ and $J_{3}$ are the corresponding principal centroidal bending inertial moments of the cross-section.

\subsection{Force and torque-driven flexible beam in a helicoidal motion}

This example was presented by Ibrahimbegović and Al Mikdad [13]. The straight beam with the centroidal axis in the $X$ direction is at its left edge constrained to slide along and rotate about the horizontal $Z$ axis. At the same edge, the torque, $M_{Z}$, and the force, $F_{Z}=0.05 \cdot M_{Z}$, are applied simultaneously, see Fig. 2, where the time-dependence of $M_{Z}$ is also depicted. Geometrical and material properties of the beam are taken from [13]: $L=10$, $A \rho=1, E A=G A=10^{4}, E J=G J=10^{3}$ and $\boldsymbol{J}_{\rho}=\operatorname{diag}\left[\begin{array}{lll}20 & 10 & 10\end{array}\right]$. 


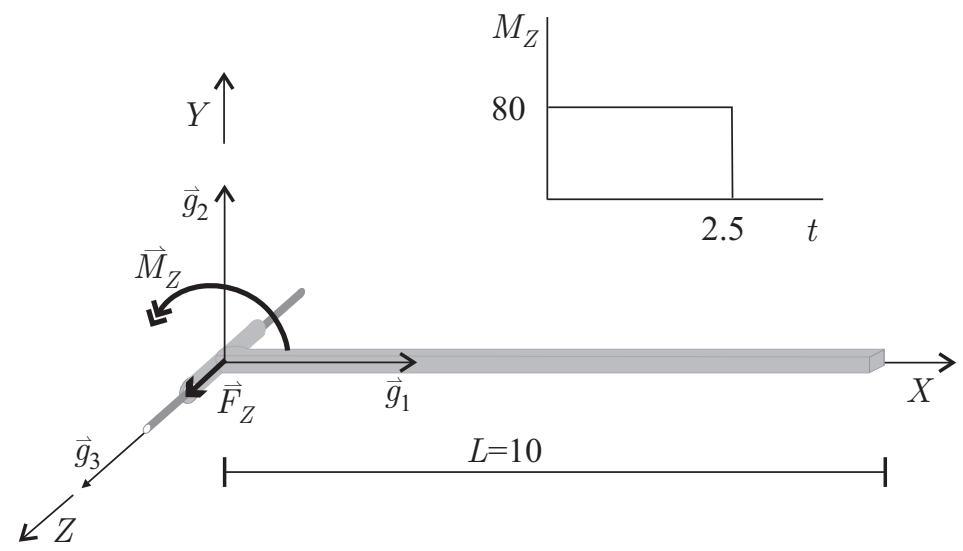

Fig. 2. Force and torque-driven flexible beam.

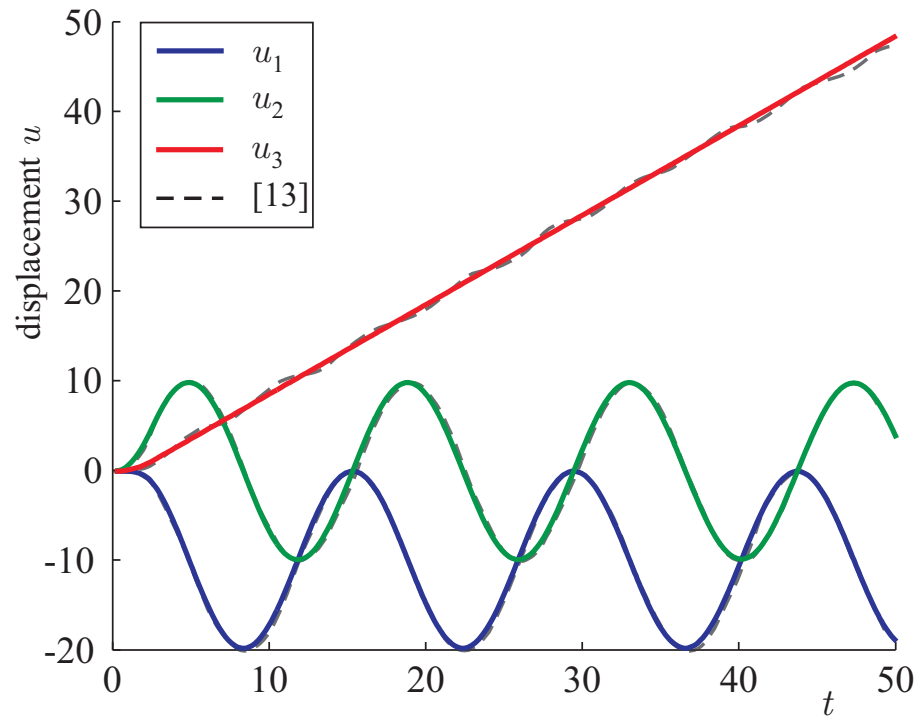

Fig. 3. Comparison of free-end displacements for the force and torque-driven flexible beam; two linear elements.

The boundary values of the components of the rotational quaternions and quaternion velocities at $x=0$ are: $q_{2}(0, t)=q_{3}(0, t)=0$ and $\lambda_{2}(0, t)=$ $\lambda_{3}(0, t)=0$. The initial values of the first components of the rotational quaternions of the beam axis are taken to be one, $q_{0}(x, 0)=1$, the rest of the components being zero. We analyze the behaviour of the beam in the time interval $[0,50]$. Two linear elements with the two-point integration rule were applied. The computational tolerances were chosen as: the maximum local absolute error $\varepsilon_{\mathrm{abs}}=10^{-2}$ and the local relative error $\varepsilon_{\text {rel }}=10^{-1}$. These tolerances required 399 time steps with the time steps varying from 0.0195 to 0.348 . The variations of the free-end displacements $u_{1}, u_{2}, u_{3}$ with time are displayed in 

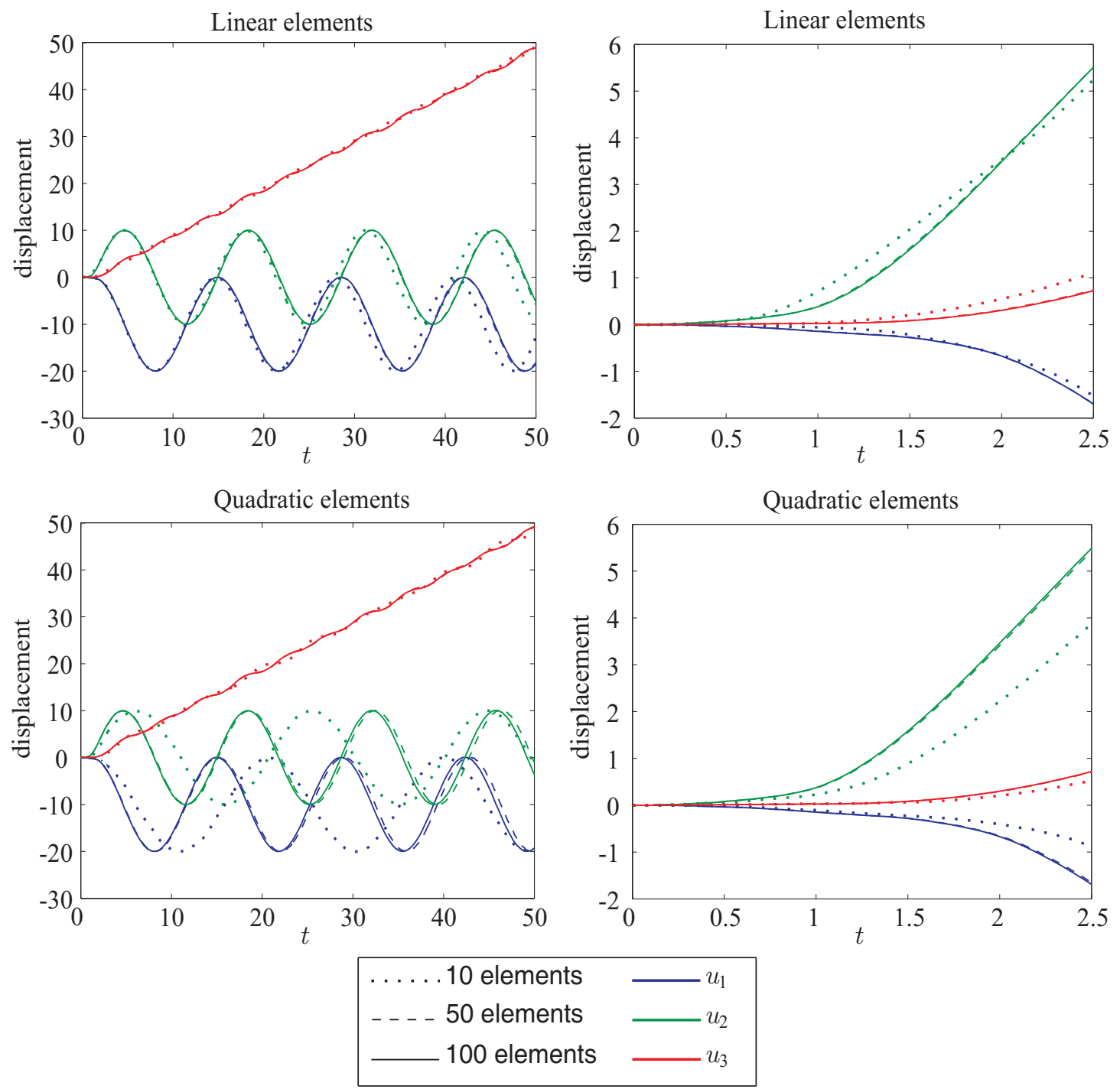

Fig. 4. The force and torque-driven flexible beam: displacements in time intervals $[0,50]$ (left) and $[0,2.5]$ (right).

Fig. 3, and the results compared with [13]. An excellent agreement between the two results can be observed.

It is instructive to analyze further the accuracy of the solution as a function of the number and the degree of interpolation. The computational tolerances are now taken to be more strict: $\varepsilon_{\text {abs }}=10^{-3}$ for all unknowns; $\varepsilon_{\text {rel }}=10^{-3}$ for the displacements and rotational quaternions, and $\varepsilon_{\text {rel }}=10^{-1}$ for the velocities and quaternion velocities. Having higher tolerances for the velocities results in lower computational times without decreasing the accuracy of the primary unknowns. The results for 10, 50 and 100 linear and quadratic elements with 
the 5- and 6-point Gauss integration rule are shown in Fig. 4. Both linear and quadratic elements approach to the same solution with the increasing number of elements. It is interesting to compare the results of the coarse mesh consisting of 10 elements with the results of the 100-element solution in some more detail. It is clear that the results of the 10 linear element mesh nearly coincide with those of the 50 and 100 element meshes. This is not the case, however, for the model with ten quadratic elements, which exihits much slower oscillations as required. In fact, neither the overall global nor the short-term local dynamic response of the beam is described sufficiently well. If the number of quadratic elements is sufficiently increased, however, the results of the linear and quadratic elements converge to the same solution.

\subsection{Flexible beam with prescribed velocities}

By this example we wish to demonstrate how the present approach is capable of considering essential boundary conditions. The geometrical and material data of the beam are taken to be identical to the previous example. The left edge of the beam is fixed in such a way that the displacements in the $X$ and $Y$ directions and the rotations about the $X$ and $Y$ directions together with the corresponding velocities are zero. The velocities in the $Z$ direction and the angular velocities about the $Z$ direction are prescribed as shown in Fig. 5. For the comparison reasons, the prescribed velocities (solid line) are given as the bilinear approximation of the numerical solution of the previous example, and are given by

$$
\dot{u}_{3}=\left\{\begin{array}{c}
0.4036 t 0 \leq t<2.5 \\
1.009 \quad t \geq 2.5
\end{array} \quad \dot{\vartheta}_{3}=\left\{\begin{array}{cc}
0.1818 t & 0 \leq t<2.5 \\
0.4545 & t \geq 2.5
\end{array} .\right.\right.
$$

The mesh of two linear elements was applied. The computational tolerances were chosen as $\varepsilon_{\text {abs }}=\varepsilon_{\text {rel }}=10^{-1}$.

In Fig. 5 we compare the time variations of the free-end displacements of the force and torque-driven beam from the previous example (solid line) with the present beam having the prescribed left-edge velocities as described above (dots). We can observe an excellent agreement of the solutions. Minor discrepancies stem from the fact that the enforced velocities were only an approximation of those in the solution of the force and torque-driven beam. 

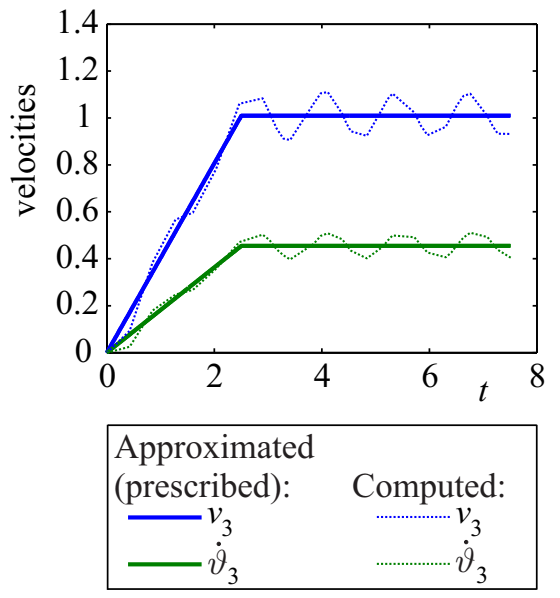

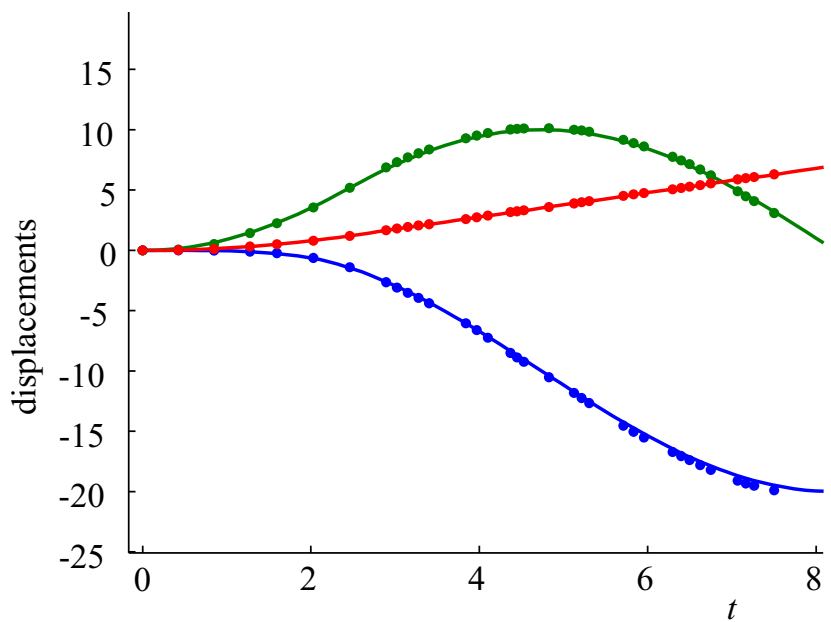

Fig. 5. Trajectory of left-edge velocities and comparison of free-end displacements.

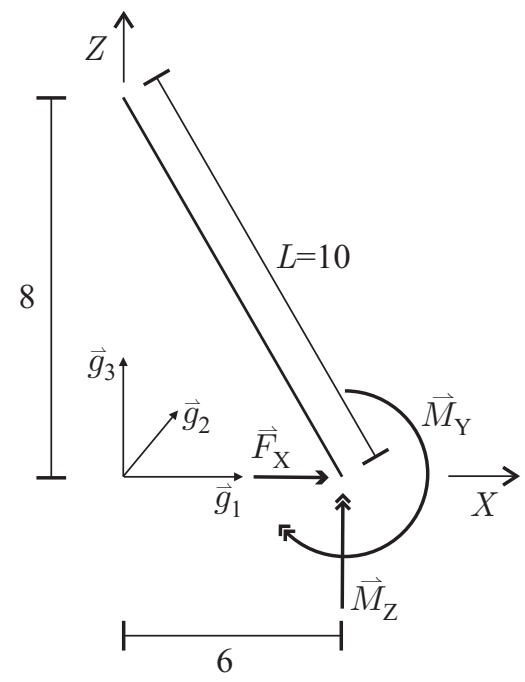

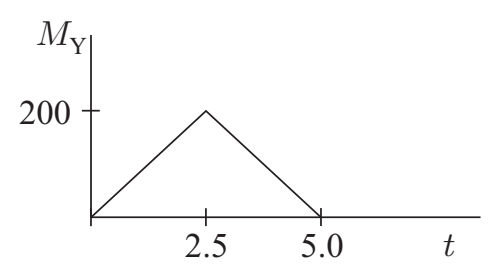

$F_{X}(t)=M_{\mathrm{Y}}(t) / 10$

$M_{\mathrm{Z}}(t) / 10=M_{\mathrm{Y}}(t) / 2$

Fig. 6. Free-free flexible beam.

\subsection{Free-free flexible beam}

The free-free flexible beam undergoing large overall spatial motion was first analyzed by Simo and Vu-Quoc [30]. The free beam is initially simultaneously subjected to the force and the torque (Fig. 6). The remaining data of the beam are as in [30]:

$$
\begin{aligned}
L & =10 \quad A \rho=1 \\
E A & =G A=10^{4} \quad E J=G J=500 \\
\boldsymbol{J}_{\rho} & =\operatorname{diag}\left[\begin{array}{lll}
10 & 10 & 10
\end{array}\right] .
\end{aligned}
$$


As in [30] the finite-element mesh consists of 10 linear elements. The sevenpoint Gaussian integration rule is applied. The computational tolerances were defined with the maximum local absolute error $\varepsilon_{\text {abs }}=10^{-3}$ for all unknowns; the maximum local relative error $\varepsilon_{\text {rel }}=10^{-3}$ for the displacements and the rotational quaternions, and $\varepsilon_{\text {rel }}=10^{-1}$ for the velocities. With such tolerances being assumed, 155 time steps were needed with the time steps varying from 0.068 to 0.208 . This number of time steps is comparable with those in [30], where 150 equal time steps were used.

For the fair comparison with Simo and Vu-Quoc [30], the results were obtained also at the predefined times using the property of the standard Matlab ode routines. Firstly the results for the fixed time steps 0.5 over the whole time interval were computed, see Fig. 7. In the next stage of the comparison, the results at the predefined time instants within the shorter time intervals $[0,7]$ and $[0,4.5]$ were computed. These particular time instants, marked also in Figs. 8 and 9, coincide with those of Simo and Vu-Quoc in [30, Figs. 17, 19]. Fig. 7 shows the three dimensional plot of the deformed beam and the trajectories of its edges. Figs. 8 and 9 show the projections of the deformed beam onto the coordinate planes. The present results again compare well with the results displayed in [30].

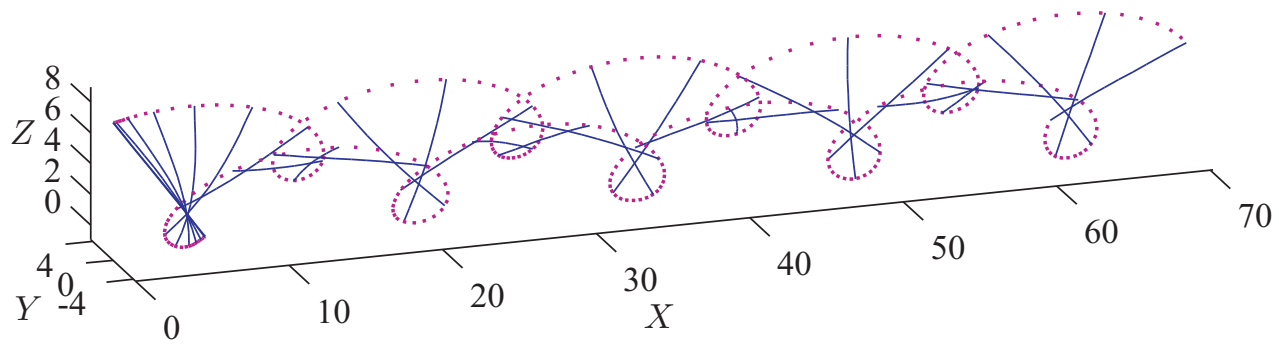

Fig. 7. Axonometric view of deformed shapes; time step 0.5 , time interval $[0,15]$.

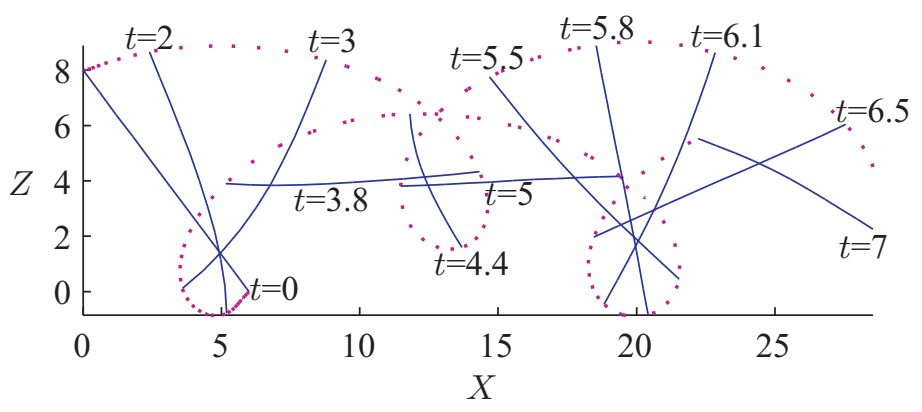

Fig. 8. Projection of the deformed shapes on the coordinate plane XZ; time interval $[0,7]$. 


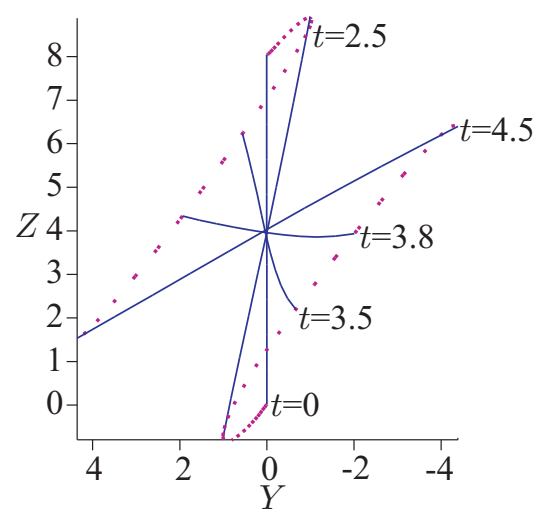

Fig. 9. Projection of the deformed shapes on the coordinate plane ZY; time interval $[0,4.5]$.

\subsection{Cantilever under distributed loads}

Highly flexible beam-like structurs often require the consideration of nonconservative, the follower-type of loads (see, e.g. Kapania and Li [19], [20], Kondoh and Atluri [21], Jiang and Olson [18] ). This example will demonstrate that the present approach can consider the follower loads properly. The analysis is carried out for the cantilever beam subjected, respectively, to two different types of uniformly distributed loads: (i) conservative and (ii) follower ones (Fig. 10). The following data of the beam have been adopted:

$$
\begin{aligned}
& \rho A_{1}=0.2 \\
& E J_{2}=E J_{3}=2.5 \cdot 10^{6} \quad E A_{1}=3 \cdot 10^{5} \\
& G A_{2}=G A_{3}=2.4 \cdot 10^{5} \quad G J_{1}=5 \cdot 10^{6} \\
& \boldsymbol{J}_{\rho}=\frac{1}{12} \cdot 10^{4} \operatorname{diag}\left[\begin{array}{lll}
2 & 1 & 1
\end{array}\right] \text {. }
\end{aligned}
$$
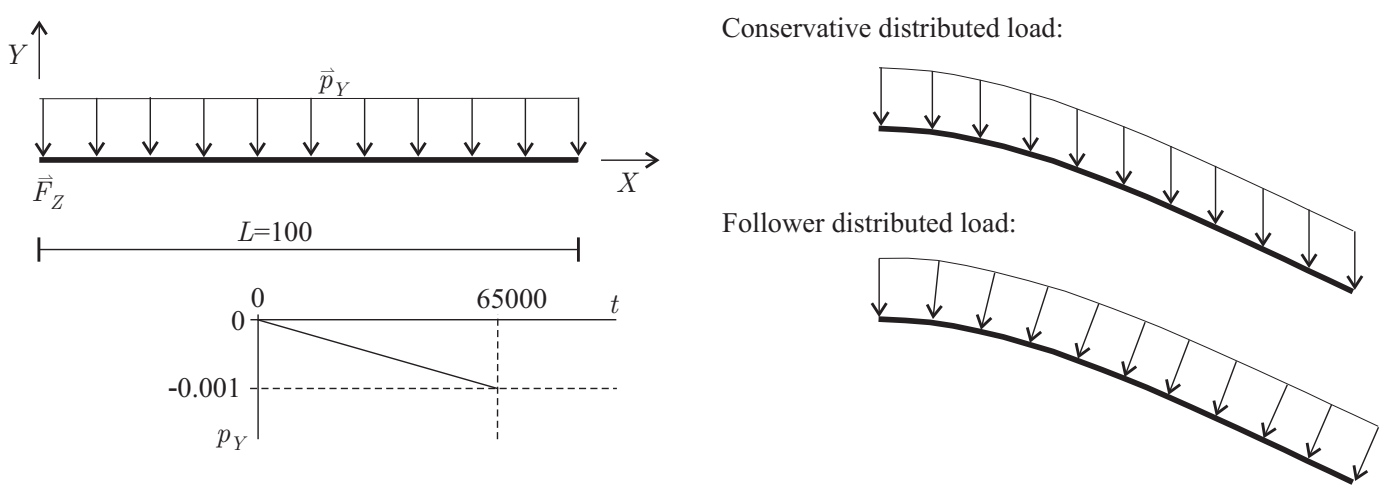

Fig. 10. Cantilever under uniformly distributed loads. 
The load is applied very slowly to show that the present formulation is able to simulate a static response in a dynamic manner (see Fig. 10). The finiteelement mesh of 10 quadratic elements was used. The computational tolerances were $\varepsilon_{\mathrm{abs}}=\varepsilon_{\mathrm{rel}}=10^{-3}$. Fig. 11 shows the deformed shapes at several discrete times and normalized loads $\lambda=\frac{p_{Y} L^{3}}{E J_{3}}$. Fig. 12 shows the normalized tip displacements vs. time.

Conservative distributed load:

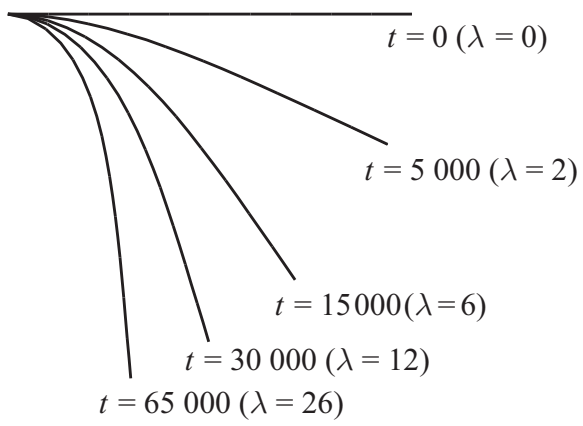

Follower distributed load:

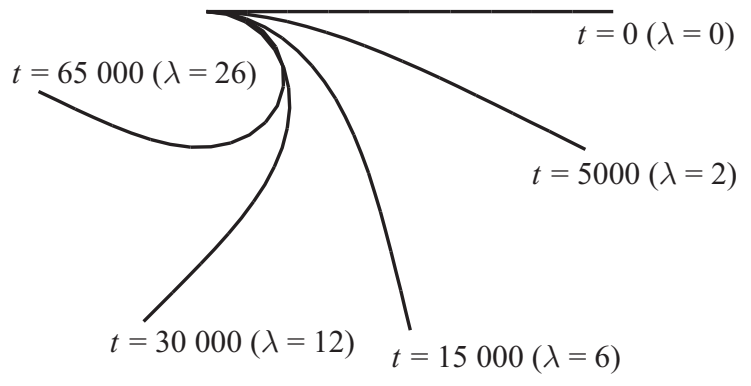

Fig. 11. Cantilever under uniformly distributed loads. Deformed shapes.

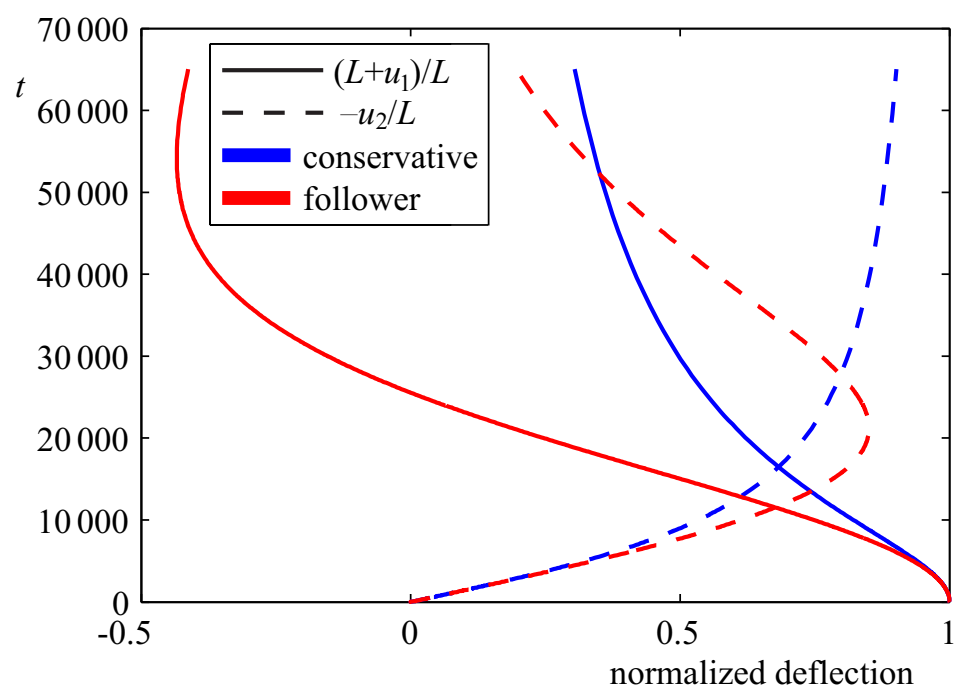

Fig. 12. Normalized tip deflections of a cantilever beam.

The present result are in excellent agreement with the ones presented in [20], $[21]$ and [18] that were obtained by the static analysis. 


\subsection{Hinged beam}

The example of the dynamic behaviour of a straight hinged beam was first presented and analyzed by Bauchau and Theron [2] and later on by Bottasso and Borri [4] to demonstrate the advantages of the energy-preserving and energy-decaying integration schemes; see also the energy-conserving schemes by [12], [28], [31], [32]. Here we demonstrate the ability of the proposed method to compute the results without setting any constraint on conserving/decaying of the energy. We also study how the density of the mesh and the local error tolerances influence the accuracy of the computed internal forces and show that we can compute the adequate response spectrum.

The $2.4 \mathrm{~m}$ long straight beam with its axis in the direction of vector $\vec{g}_{1}$ is hinged at the root so that only the rotation about the vector $\vec{g}_{2}$ is allowed. The other end is free. The geometry of the beam and the loading data are presented in Fig. 13. The remaining properties of the beam are

$$
\begin{aligned}
E A_{1} & =4.35080 \cdot 10^{7} \mathrm{~N} & & \\
G A_{2} & =1.40385 \cdot 10^{7} \mathrm{~N} & & \\
G A_{3} & =2.80769 \cdot 10^{6} \mathrm{~N} & & \rho A_{1}=1.60920 \mathrm{~N} / \mathrm{m} \\
G J_{1} & =2.80514 \cdot 10^{4} \mathrm{Nm}^{2} & & \rho J_{1}=1.19092 \cdot 10^{-2} \mathrm{~kg} \mathrm{~m} \\
E J_{2} & =2.32577 \cdot 10^{4} \mathrm{Nm}^{2} & & \rho J_{2}=8.60200 \cdot 10^{-4} \mathrm{~kg} \mathrm{~m} \\
E J_{3} & =2.98731 \cdot 10^{4} \mathrm{Nm}^{2} & & \rho J_{3}=1.10490 \cdot 10^{-2} \mathrm{~kg} \mathrm{~m} .
\end{aligned}
$$

The present analysis employs three meshes consisting of 4, 10 and 20 linear elements, and imposes two local error tolerances, $\varepsilon_{\text {abs }}=\varepsilon_{\text {rel }}=10^{-2}$ and $\varepsilon_{\text {abs }}=$ $\varepsilon_{\text {rel }}=10^{-3}$. The three-point Gaussian integration rule is applied. The dynamic response of the beam was computed for $t \leq 0.25$ with the number of time steps varying according to the imposed local error tolerances.

In Table 1 the number of time steps and the total angle of free-end rotation are shown and compared with [2] and [4]. Time histories of the axial force at
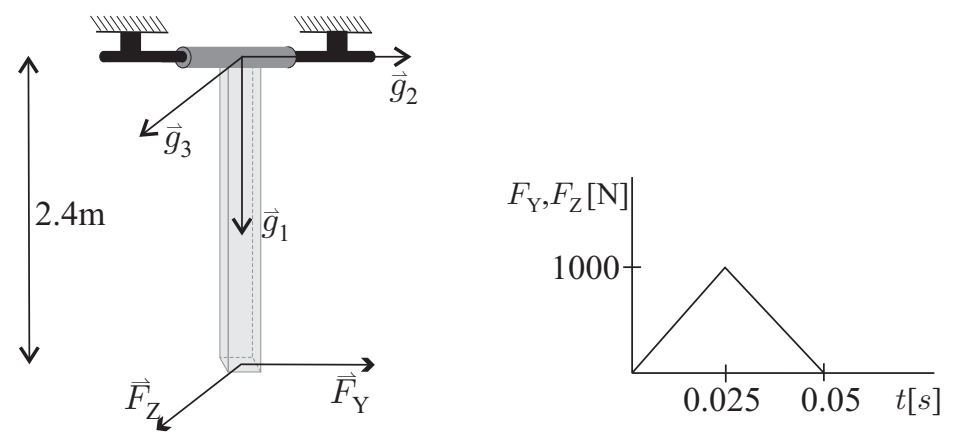

Fig. 13. Hinged beam. 
the hinge are displayed in Fig. 14. The frequency spectra performed over the time interval $t \in[0.122,0.25]$ are shown in Fig. 15. The discrete fast Fourier transform [25] (FFT) has been applied. The graphs in Fig. 14 indicate that the four-element meshes only supply a globally accurate response of axial forces if tolerance is less or equal to $10^{-3}$. An increased number of elements improves results considerably. With the use of the mesh of 20 linear elements we are able to compute accurately the axial force time response and the corresponding frequency spectrum. The results well compare with the results from [4], based on the energy-conserving integration method.

We wish to stress that the present results confirm the results from [4] that the first two modes dominate the behaviour of the axial force with time. Figs 14 and 15 further indicate that the second mode of oscilations dominantes between these two modes. We present in Fig. 15 the frequency spectrum up to $5000 \mathrm{~Hz}$. The second frequency, roughly $3384 \mathrm{~Hz}$, is well estimated for all meshes, the first frequency being about $441 \mathrm{~Hz}$ is more sensitive and can be obtained with a sufficient accuracy only by denser meshes. Note that the present results show no interfering frequencies. Note also that the frequency domain for the 128-point FFT is too small resulting in an inaccurate second frequency, and that the increase of the number of points in FFT highly increases the magnitude of the adequate frequencies $F F T^{2}\left(N_{x}\right)$, while the frequency values remain practically unchanged. For the mesh of 20 linear elements, the convergence of the first two frequencies of the axial force of the hinged beam with the number of points used in the FFT algorithm is presented in Table 2. It is clear that the convergence is almost independent on the prescribed local error tolerance of the solver.

Table 1

Number of time steps and total angle of rotation at free end.

\begin{tabular}{c|c|c|c|c} 
element & num. of el. & tolerances & number of time steps & total angle $\left[^{\circ}\right]$ \\
\hline present & 4 & $10^{-2}$ & 7692 & 103.3 \\
present & 10 & $10^{-2}$ & 24082 & 104.0 \\
present & 20 & $10^{-2}$ & 39993 & 104.0 \\
present & 4 & $10^{-3}$ & 26858 & 103.3 \\
present & 10 & $10^{-3}$ & 69513 & 104.0 \\
present & 20 & $10^{-3}$ & 117810 & 104.0 \\
{$[2]$ cubic } & 4 & - & $250-32000$ & 104.2 \\
{$[4]$} & 10 & - & 250 and 1000 & -
\end{tabular}



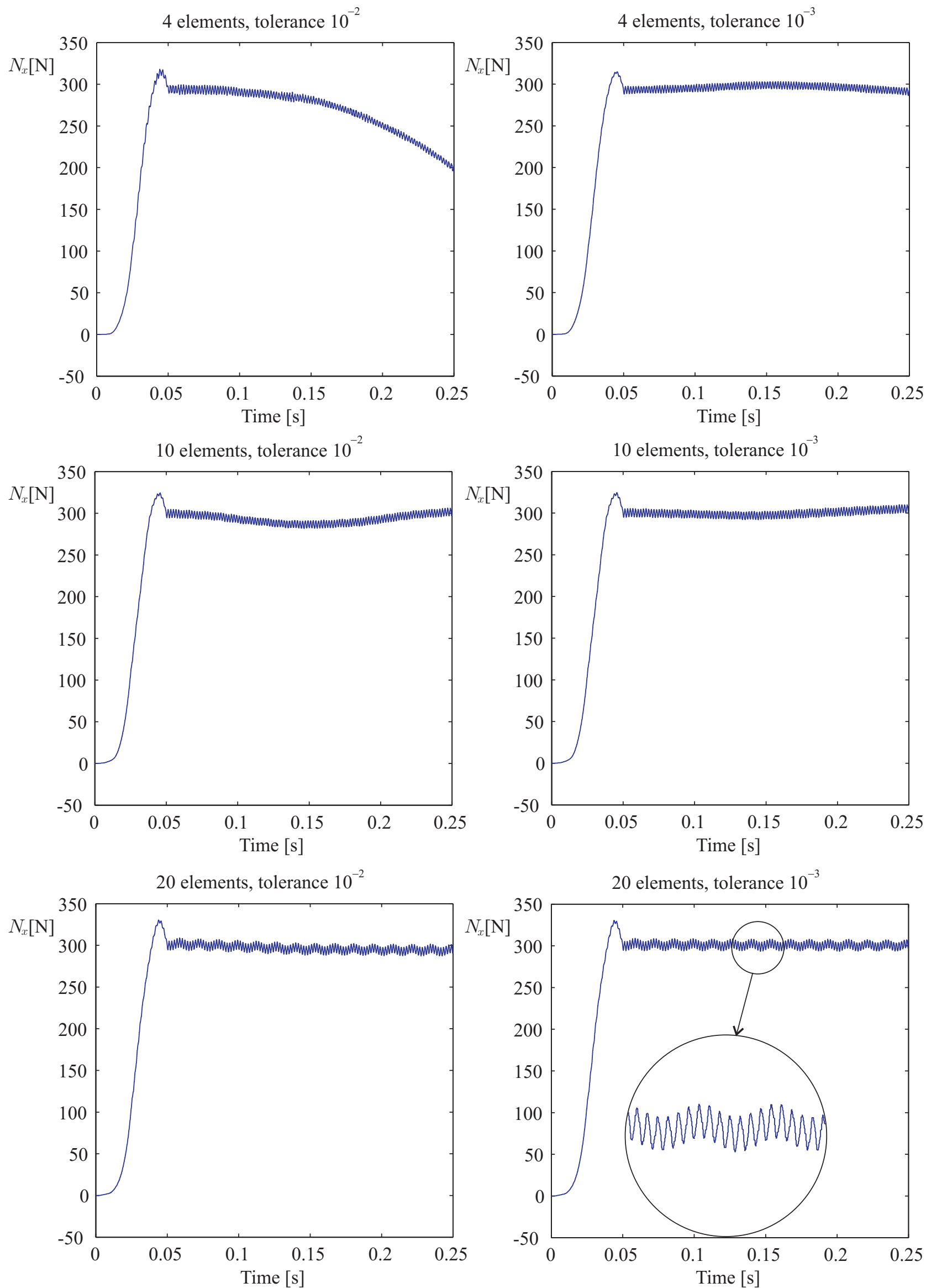

Fig. 14. Graphs of the axial force, $N_{x}$, versus time at the hinge. The effects of the number of linear elements and the error tolerances. 

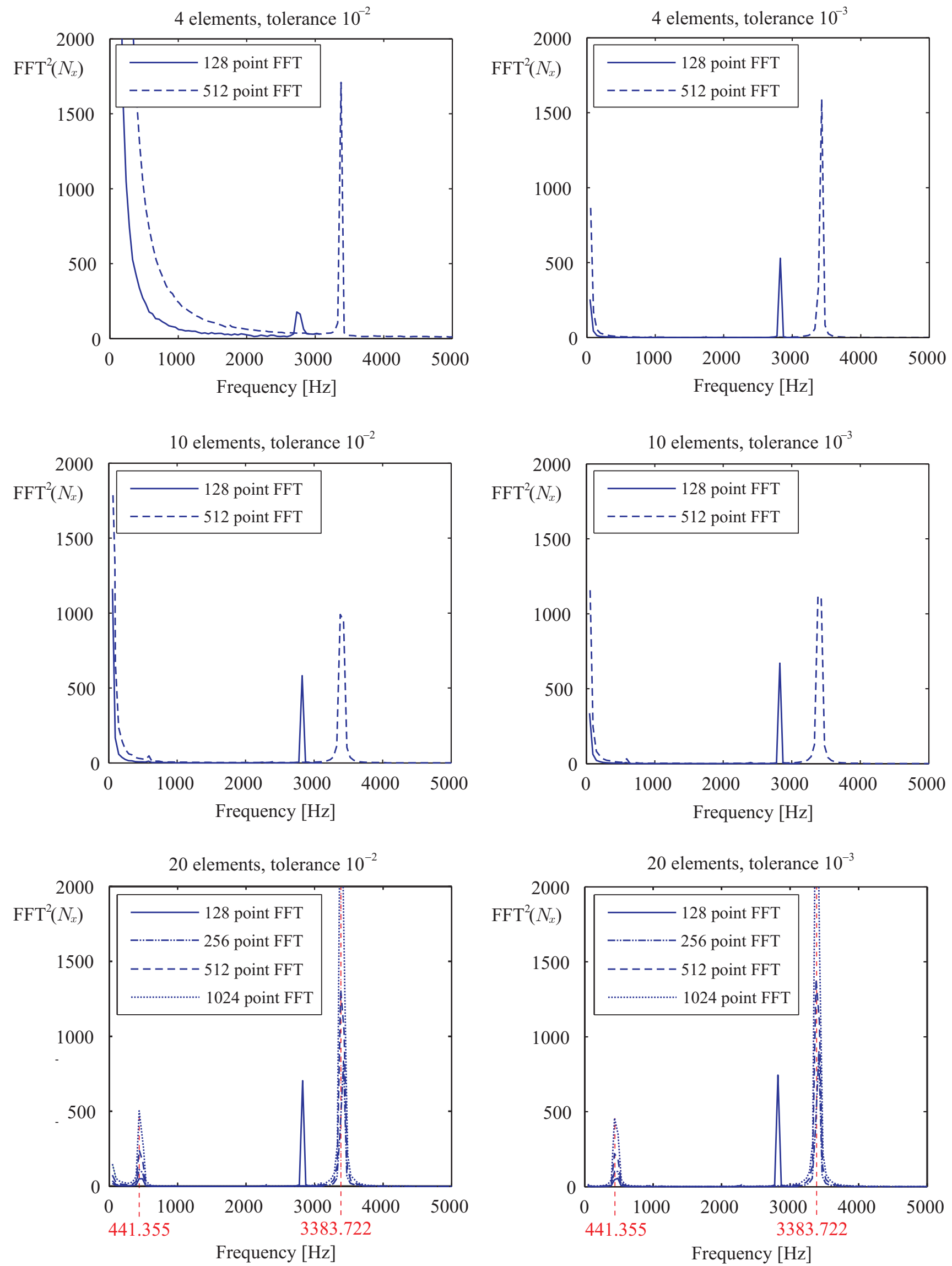

Fig. 15. Frequency spectra. The effects $27_{\text {of }}$ the number of linear elements, error tolerances, and the number of discrete points in FFT. 
Table 2

Convergence of the frequencies in the FFT algorithm. 20 element mesh.

\begin{tabular}{c|c|c|c|c} 
& \multicolumn{2}{|c|}{$1^{\text {st }}$ frequency } & \multicolumn{2}{c}{$2^{\text {nd }}$ frequency } \\
\hline$N_{\text {FFT }}$ & $\varepsilon=10^{-2}$ & $\varepsilon=10^{-3}$ & $\varepsilon=10^{-2}$ & $\varepsilon=10^{-3}$ \\
\hline 128 & 487.014 & 487.044 & 2824.856 & 2824.682 \\
\hline 256 & 440.060 & 440.928 & 3422.695 & 3422.695 \\
\hline 512 & 440.901 & 440.928 & 3380.450 & 3380.243 \\
\hline 1024 & 441.355 & 441.355 & 3383.722 & 3383.722 \\
\hline
\end{tabular}

$N_{\mathrm{FFT}}=$ number of points for discrete $\mathrm{FFT}$

$\varepsilon=$ local absolute and relative error tolerance

\section{CONCLUSIONS}

The paper presents a new finite element approach for the dynamic analysis of the geometrically exact three-dimensional beam-like structures based on the rotational quaternion. We completely abandon the matrix representation of rotations combined with the rotational vector and present the equations for the dynamic analysis in the quaternion description. The collocation type of discretization is used and the novel form of the boundary condition that avoids differential-algebraic system of equations is presented. The proposed numerical implementation of the three-dimensional beam model can successfully deal with a most demanding fully spatial dynamic behaviour of the beam-like structures. The governing equations are written in the 4-dimensional linear space of quaternions and are thus suitable to be solved by the standard RungeKutta solvers. This is in sharp contrast to rotational vector approach which is inherently related to the special orthogonal group $S O(3)$ and is hence not appropriate for the standard Runge-Kutta methods. Since the standard integrator can now be used, the update procedure for the rotational parameters needs no extra treatment. By the direct use of the Matlab ode23tb solver we benefit from theoretically well based adaptive time-step algorithm and avoid the analytical linearization of the governing equations. The main advantage of the proposed method is thus in its ability to use a wide range of modern solvers for stiff differential equations that have been derived for linear configuration spaces. Another advantage is in the direct use of the quaternion algebra, which has been proven to be an efficient tool in numerical algorithms. Yet the present paper introduces only one of a variety of quaternion-based approaches. Such a completely new approach can be a challenge and an inspiration for a further research, as the method can be further developed in many aspects. 


\section{References}

[1] Argyris, J.H. An excursion into large rotations. Comput. Methods Appl. Mech. Engrg. 32 (1982) 85-155.

[2] Bauchau, O.A., Theron, N.J. Energy decaying scheme for non-linear beam models. Comput. Methods Appl. Mech. Engrg. 134 (1996) 37-56.

[3] Betsch, P., Steinmann, P. Frame-indifferent beam finite elements based upon the geometrically exact beam theory. Int. J. Numer. Meth. Eng. 54 (2002) 17751788.

[4] Bottasso, C.L., Borri, M. Energy preserving/decaying schemes for nonlinear beam dynamics using the helicoidal approximation. Comput. Methods Appl. Mech. Engrg. 143 (1997) 393-415.

[5] Butcher, J.C. The Numerical Analysis of Ordinary Differential Equations. Runge-Kutta and General Linear Methods. John Wiley \& Sons, ChichesterNew York-Brisbane-Toronto-Singapore, 1987.

[6] Ghosh, S., Roy, D. Consistent quaternion interpolation for objective finite element approximation of geometrically exact beam. Comput. Methods Appl. Mech. Engrg. 198 (2008) 555-571.

[7] Hairer, E., Wanner, G. Solving Ordinary Differential Equations II. Stiff and Differential-Algebraic Problems. Springer-Verlag, Berlin-Heidelrberg-New York, 1991.

[8] Hosea, M.E., Shampine, L.F. Analysis and implementation of TR-BDF2. Appl. Num. Math. 20 (1996) 21-37.

[9] Ibrahimbegović, A. On FE implementation of geometrically nonlinear Reissner's beam theory: Three-dimensional curved beam elements. Comput. Methods Appl. Mech. Engrg. 122 (1995) 11-26.

[10] Ibrahimbegović, A. On the choice of finite rotation parameters. Comput. Methods Appl. Mech. Engrg. 149 (1997) 49-71.

[11] Ibrahimbegović, A., Frey, F., KožAr, I. Computational aspects of vectorlike parametrization of three-dimensional finite rotations. Int. J. Numer. Methods Engng. 38 (1995) 3653-3673.

[12] Ibrahimbegović, A., Mamouri S. Energy conserving/decaying implicit timestepping scheme for nonlinear dynamics of three-dimensional beams undergoing finite rotations. Comput. Methods Appl. Mech. Engrg. 191 (2002) 4241-4258.

[13] Ibrahimbegović, A., Al Mikdad, M. Finite rotations in dynamics of beams and implicit time-stepping schemes. Int. J. Numer. Meth. Engng. 41 (1998) 781-814.

[14] Ibrahimbegović, A., TAYlor R. L. On the role of frame-invariance of structural mechanics models at finite rotations. Comput. Methods Appl. Mech. Engrg. 191 (2002) 5159-5176. 
[15] Iura, M., AtLuri, S.N. On a consistent theory and variational formulation of finitely stretched and rotated 3-D space-curved beams. Comput. Mech. 4 (1989) $73-88$.

[16] Jelenič, G., SaJe, M. A kinematically exact space finite strain beam modelfinite element formulation by generalized virtual work principle. Comput. Methods Appl. Mech. Engrg. 120 (1995) 131-161.

[17] Jelenič, G., CRisfield, M.A. Geometrically exact 3D beam theory: implementation of a strain-invariant finite element for statics and dynamics. Comput. Methods Appl. Mech. Engrg. 171 (1999) 141-171.

[18] JiAng, J., Olson, M.D. Large elastic-plastic deformations of slender beams: Co-rotational theory vs. von Karman theory. Comput. Mech. 15 (1994) 117-128.

[19] Kapania, R. K., LI, J. On a geometrically exact curved/twisted beam theory under rigid cross-section assumption. Comput. Mech. 30 (2003) 428-443.

[20] Kapania, R. K., Li, J. A formulation and implementation of geometrically exact curved beam elements incorporating finite strains and finite rotations. Comput. Mech. 30 (2003) 444-459.

[21] Kondoh K., Atuuri S.N. Large-deformation, elasto-plastic analysis of frames under nonconservative loading, using explicitly derived tangent stiffnesses based on assumed stresses. Comput. Mech. 2 (1987) 1-25.

[22] LANG H., Linn J., ARnold M. Multi-body dynamics simulation of geometrically exact Cosserat rods. Multibody Syst. Dyn. 25 (2011) 285-312.

[23] Lim H., TAYLOR R.L. An explicit-implicit method for flexible-rigid multibody systems. Finite Elem. Anal. Des. 37 (2001) 881-900.

[24] McRobie, F.A., Lasenby, J. Simo-Vu Quoc rods using Clifford algebra. Int. J. Numer. Methods Eng. 45 (1999) 377-398.

[25] The MathWorks, Inc. MATLAB, Using MATLAB. Natick, http://www. mathworks.com, 1999.

[26] Poreous, I.R. Clifford Algebras and the Classical Groups. Cambridge University Press, Cambridge, 1995.

[27] Qilin Z., Jun J., Zhenghua H., Zhiguo C. Analytical mechanics solution for mechanism motion and elastic deformation hybrid problem of beam system. Finite Elem. Anal. Des. 45 (2009) 901-907.

[28] Romero, I., Armero, F. An objective finite element approximation of the kinematics of geometrically exact rods and its use in the formulation of an energy-momentum conserving scheme in dynamics. Int. J. Numer. Methods Eng. 54 (2002) 1683-1716.

[29] Simo, J.C., Vu-Quoc, L. On the dynamics in space of rods undergoing large motions - A geometrically exact approach. Comput. Methods Appl. Mech. Engrg. 66 (1988) 125-161. 
[30] Simo, J.C., Vu-Quoc, L. A three-dimensional finite-strain rod model. Part II: Computational aspects. Comput. Methods Appl. Mech. Engrg. 58 (1986) 79-116.

[31] Simo, J.C., Wong, K.K. Unconditionally stable algorithms for rigid body dynamics that exactly preserve energy and momentum. Int. J. Numer. Methods Engng. 31 (1991) 19-52.

[32] Simo, J.C., Tarnow, N., Doblare, M. Non-linear dynamics of threedimensional rods: exact energy and momentum conserving algorithms. Int. J. Numer. Methods Engng. 38 (1995) 1431-1473.

[33] Ward, J.P. Quaternions and Cayley Numbers. Kluwer Academic Publishers, Dordrecht-Boston-London, 1997.

[34] Zupan, D., Saje, M. A new finite element formulation of three-dimensional beam theory based on interpolation of curvature. Comput. Model. Eng. Sci. 4, 2 (2003) 301-318.

[35] Zupan, D., SAJE, M. Convergence properties of materially and geometrically non-linear finite-element spatial beam analysis. Finite Elem. Anal. Des. 45 (2008) 60-69.

[36] Zupan, E., SaJe, M. Integrating rotation from angular velocity. Adv. Eng. Softw. 42 (2011) 723-733.

[37] Zupan, E., SAJE, M., Zupan, D. The quaternion-based three-dimensional beam theory. Comput. Methods Appl. Mech. Engrg. 198 (2009) 3944-3956. 